\title{
Combinatorial stability of non-deterministic systems
}

\author{
P. Duarte ${ }^{1}$, M. J. Torres ${ }^{2}$ \\ 1 CMAF, Faculdade de Ciências da Universidade de Lisboa, Campo Grande, Edifício CS, \\ 1749-016 Lisboa, Portugal (e-mail: pduarte@ptmat.fc.ul.pt) \\ ${ }^{2}$ CMAT, Departamento de Matemática, Universidade do Minho, Campus de Gualtar, 4700- \\ 057 Braga, Portugal (e-mail: jtorres@math.uminho.pt)
}

\begin{abstract}
We introduce and study, from a combinatorial-topological viewpoint, some semigroups of continuous non-deterministic dynamical systems. Combinatorial stability, i.e. the persistence of the combinatorics of the attractors, is characterized and its genericity established. Some implications on topological (deterministic) dynamics are drawn.
\end{abstract}

\section{Introduction}

In the framework of smooth deterministic dynamical systems, i.e. smooth maps, diffeomorphisms and flows on finite-dimensional compact manifolds, it has long been belived that typical dynamical systems should exhibit a finite number of attractors, their basins of attraction covering almost everything.

Smale's program in the early 1960s aimed to prove the (topological) genericity of structurally stable systems. See, e.g., [Sm]. The concept of structural stablity was introduced in the 1930s by Andronov-Pontryagin. It means that under small perturbations the dynamics are topologically equivalent to the original system. Although Smale's program was proved to be wrong one decade later, it played a fundamental role in the development of the theory of dynamical systems. It led to the construction of Hyperbolic Theory, studying uniform hyperbolicity and characterizing structural stability as being essentially equivalent to uniform hyperbolicity. Of course the scenario above is true for hyperbolic (Axiom A) systems. 
More recently Palis conjectured $[\mathrm{P}]$ that every dynamical system can be approximated by one with finitely many attractors, each having a stochastically stable physical measure, whose basins of attraction cover almost every point in state space. See, e.g., [V2]. A physical measure of an attractor is one that describes the system time average for a positive volume set of initial states. The set of all such initial states is called the physical measure basin of attraction. A measure on an attractor is said to be stochastically stable if it is stable under small stochastic perturbations of the deterministic system. More precisely, introducing a random noise, the limit measures of the random perturbations approach the attractor physical measure as the noise level tends to zero. See, e.g., [V1]. The key idea of introducing a random noise in a deterministic system, and then looking at the limit measures as the noise level tends to zero, is usually attributed to Kolmogorov. Under very mild conditions, a random noise can have a powerfull simplifying effect on the complexity of the dynamics of a deterministic system. Namely, under arbitrary small random perturbations any deterministic system has finitely many attractors (see, for instance, $[\mathrm{A}]$ ). Another simplifying effect may be seen on the Perron-Frobenius operator, whose fixed points are precisely the system invariant measures. In general, the spectrum of this linear operator, reflecting the action of dynamics upon measures supported in state space, can be complex, but when a random noise is turned on this usually makes the operator compact or quasi-compact with pure point spectrum. A compact operator can be, spectrally speaking, well approximated by finite-dimension operators. Thus random perturbations of a deterministic system may, just as well, be considered on finite (discrete) approximations of state space. Finite state Markov chains are the stochastic, or random dynamical systems on a finite state space. One may think that these dynamical systems are what we actually see when running computer simulations of deterministic dynamical systems. Each such dynamical system is specified by a stochastic matrix with the state probability transitions. The stochastic matrix is the Perron operator of this finite state system. The Markov chain also determines an oriented graph, encapsulating some qualitative aspects of the system behaviour. The theory of finite state Markov chains establishes a correspondence between spectral properties of the stochastic matrix on one side, and combinatorial properties of the corresponding graph on the other hand. See, e.g., [B].

This theory can be nicely extended into a theory for continuous, or lower semicontinuous, random dynamical systems of Markov type on a compact manifold $X$. In the present article we develop such a theory in its topological and combinatorial aspects. Then in a complementary paper we will adress the correspondent spectral 
theory and its relation with the results obtained here. The main novelty with respect to finite state Markov chain theory is that in this context, because we are dealing with continuous systems, it makes sense defining stability: combinatorial stability or spectral stability. The core of the present article will be to establish and characterize combinatorial stability.

We define a non-deterministic dynamical system on a state space $X$ to be any multivalued mapping $\varphi: X \rightarrow \mathcal{P}(X)$, where $\mathcal{P}(X)$ denotes the set of all $X$ subsets. See [AF] for an overview on multi-valued analysis. Here the state space $X$ will be a compact manifold and we make a fundamental assumption on the state transition sets $\varphi(x)$ $(x \in X)$. They are always non-empty connected open sets. The lower semi-continuity of a non-deterministic dynamical system $\varphi$ with respect to the Hausdorff distance is then equivalent to the openness of $\operatorname{graph}(\varphi)$. Such systems will be refered throughout this work as open maps.

In $\S 3$ we define several topological spaces of open sets. See $[N]$ for an overview on topological spaces of sets. Then in $\S 4$ we introduce and topologize some semigroups of open maps. Any open map $\varphi$ can be identified with its graph $\operatorname{graph}(\varphi)$ and, therefore, seen as an open set in $X \times X$. Thus, semigroups of open maps can be given topologies as subspaces of topological spaces of open sets. The key concept of topological semigroup of open maps, to which the main Theorems 5.3 and 5.4 apply, is defined here. Examples of topological semigroups of open maps are given.

In $\S 5$ we define recurrence and chain-recurrence. Briefly, given any open map $\varphi$, a sequence $x_{0}, x_{1}, \cdots, x_{n}$ such that $x_{i} \in \varphi\left(x_{i-1}\right)$ for all $i=1, \cdots, n$ will be called a $\varphi$-orbit, and we will say that $x_{n}$ is a $\varphi$-iterate of $x_{0}$. If for every $\epsilon>0, y$ is a $\varphi_{\epsilon}^{*}$-iterate of $x$, where $\varphi_{\epsilon}^{*}$ is the open map whose graph is an $\epsilon$-radius ball of $\operatorname{graph}(\varphi)$, we say that $y$ is a $\varphi$-pseudo-iterate of $x$. The recurrent and chain-recurrent sets of $\varphi$ are defined respectively by $\Omega(\varphi)=\{x \in X: x$ is a $\varphi-$ iterate of $x\}$ and $R(\varphi)=\{x \in X: x$ is a $\varphi$-pseudo-iterate of $x\}$. Both these sets split into equivalence classes, each class being formed by states which are accessible from each other. The set of all these classes is then partially ordered by the dynamics of $\varphi$. At the bottom of this hierarchy are two special limit sets: the final recurrent and the final chainrecurrent sets, denoted respectively by $\Omega_{\text {final }}(\varphi)$ and $R_{\text {final }}(\varphi)$, of all states $x \in \Omega(\varphi)$ $\left(x \in R_{\text {final }}(\varphi)\right)$ such that every iterate (pseudo-iterate) of $x$ still has some iterate (pseudo-iterate) which comes back to $x$. These limit sets contain all the asymptotic dynamical behaviour of $\varphi$. They both decompose into a finite number of connected pieces which are permuted by $\varphi$. The restriction of $\varphi$ to each of these pieces being in 
some sense irreducible. See Theorems 5.1 and 5.2.

We say that two open maps $\varphi$ and $\psi$ are combinatorially equivalent if and only if there is a bijective conjugation between both maps' actions on the final recurrent sets' connected components. Given a topological semigroup of open maps $\mathcal{O}_{1}$, an open map $\varphi \in \mathcal{O}_{1}$ will be called $\mathcal{O}_{1}$-combinatorially stable when there is a neighbourhood of $\varphi$ in $\mathcal{O}_{1}$ where all open maps are combinatorially equivalent to $\varphi$. As any iterate is also a pseudo-iterate, there is a natural relation between the connected pieces of $\Omega_{\text {final }}(\varphi)$ and those of $R_{\text {final }}(\varphi)$. In Theorem 5.3 we characterize combinatorial stability: for any topological semigroup of open maps $\mathcal{O}_{1}$ this relation is bijective if and only if $\varphi$ is $\mathcal{O}_{1}$-combinatorially stable. We also prove the genericity of combinatorial stability for topological semigroups of open maps, see Theorem 5.4. At the end of $\S 5$ we characterize the continuity of the attractors, i.e. the connected components of $\Omega_{\text {final }}(\varphi)$ or $R_{\text {final }}(\varphi)$, see Theorem 5.5. When a combinatorially stable map is a continuity point of the attractors we say that it is topologically stable. Finally, we establish, see Theorem 5.6, that (in a topological sense) any generic open map $\varphi$, in some topological semigroup of open maps $\mathcal{O}_{1}$, is topologically stable.

In the last section, $\S 6$, we drive some applications to the deterministic dynamics of continuous mappings. See [Pi] for a survey on $C^{0}$ dynamics. We will denote by $\mathcal{C}^{0}(X)$ the space of all continuous mappings $f: X \rightarrow X$ with the usual $C^{0}$ topology of uniform convergence. Given any map $f \in \mathcal{C}^{0}(X)$ we define chain recurrence and the final chain-recurrent set, $R_{\text {final }}(f)$, in a similar way as for open maps. Let $\mathcal{S} \subseteq \mathcal{C}^{0}(X)$ be a topological space or semigroup such that the inclusion $\mathcal{S} \hookrightarrow \mathcal{C}^{0}(X)$ is continuous. Let us say that $f \in \mathcal{S}$ is strongly combinatorially stable in $\mathcal{S}$ if and only if there is a neighbourhood of $f$ in $\mathcal{S}$ where all maps are combinatorially equivalent to $f$, in the sense that there is a bijective conjugation between the actions of both maps on the connected components of the final chain-recurrent sets. If $\mathcal{S}=\mathcal{C}^{0}(X)$, this concept becomes too strong: there is no strongly combinatorially stable map in $\mathcal{C}^{0}(X)$, but in general with some smoothness assumption on $\mathcal{S}$, the set of all strongly combinatorially stable maps in $\mathcal{S}$ is a non-trivial open subset of $\mathcal{S}$. In general, this open set is not dense in $\mathcal{S}$, as Newhouse examples with infinitely many sinks show. For instance, if $\mathcal{S}=\mathcal{C}^{1}(X)$ is the space of class $C^{1}$ mappings $f: X \rightarrow X$, systems with finitely many robustly transitive attractors (Axiom A in particular) are strongly combinatorially stable. Strong stability will not be addressed in the present article. Instead we shall work with a weaker concept. Given an open map $\varphi$, if $\operatorname{graph}(f) \subseteq \operatorname{graph}(\varphi)$, we write $f \prec \varphi$ and say that $f$ is subordinated to $\varphi$. We say that $f$ is weakly combinatorially 
stable if for every sufficiently small perturbation $\varphi$ of $f$, with $f \prec \varphi$, the combinatorics of their final chain-recurrent sets are the same. We will apply the name attractor, in this paper, to any connected invariant chain-transitive set $A \subseteq X$ which attracts a whole neighbourhood of $A$. In Theorem 6.1 we show that a map $f \in \mathcal{C}^{0}(X)$ is weakly combinatorially stable if and only if $f$ has finitely many attractors if and only if $R_{\text {final }}(f)$ has finitely many connected components. We should emphasize that 'weak combinatorial stability' is a really feeble concept. For instance, all volume-preserving maps are weakly combinatorially stable. By the Poincaré recurrence theorem, every volume-preserving map is chain transitive and, therefore, has the whole manifold as its unique attractor. In Theorem 6.2 we show that the set of maps $f \in \mathcal{C}^{0}(X)$ with finitely many attractors is dense in $\mathcal{C}^{0}(X)$. In view of the Palis conjecture, it would be much more interesting proving this theorem for spaces of smoother functions $\mathcal{S} \subseteq \mathcal{C}^{r}(X)$, with $r \geq 1$. Thus, in order to allow for possible generalizations we have proved an abstract result, see Theorem 6.3, saying that the same conclusion (density of maps with finitely many attractors) holds in every space $\mathcal{S} \subseteq \mathcal{C}^{0}(X)$ having the skeletonchain perturbation property. Of course we only prove that $\mathcal{S}=\mathcal{C}^{0}(X)$ satisfies this condition, but because this key property has a rather geometric nature, we hope in the future to be able to develop new perturbation techniques for proving that other spaces of smoother functions also share this property.

\section{Notation}

Throughout this work $X$ will denote a compact Riemannian manifold of dimension $n, d$ will be the geodesic distance on $X$ and $m$ will be the corresponding normalized $(m(X)=1)$ Riemannian volume. The state space of all systems that we consider will be $X$.

Given sets $U, V \subseteq X$ we will use the following notation:

(1) $B_{r}(U)$, respectively $\bar{B}_{r}(U)$, denotes the set of all points whose distance $d$ to $U$ is less than, respectively less than or equal to $r$;

(2) $\rho_{H}(U, V)=\inf \left\{r>0: U \subseteq B_{r}(V)\right.$ and $\left.V \subseteq B_{r}(U)\right\}$ denotes the Hausdorff distance between $U$ and $V$; 
(3) $\rho_{m}(U, V)=m(U \backslash V)+m(V \backslash U)$ stands for the $L^{1}$ distance between the functions $I_{U}$ and $I_{V}$, where

$$
I_{U}(x)=\left\{\begin{array}{lll}
1 & \text { if } & x \in U \\
0 & \text { if } & x \notin U
\end{array}\right.
$$

(4) $H^{n-1}(U)$ is the $(n-1)$-dimensional Hausdorff measure of $U$;

(5) $\bar{U}$ is the closure of $U$ in $X$;

(6) $U^{\circ}$ is the interior of $U$ in $X$;

(7) $\partial U$ is the topological boundary of $U$ in $X$;

(8) $U^{e}$ is the exterior of $U$ in $X$;

(9) $\widehat{U}=(\bar{U})^{\circ}$ is the interior of the closure of $U$ in $X$;

(10) $U^{c}$ is the complement of $U$ in $X$;

(11) $|\Xi|$ is the number of elements in a set $\Xi$.

Similar notations will be used on $X \times X$, where $d$ will stand for the metric

$$
d\left(\left(x_{1}, y_{1}\right),\left(x_{2}, y_{2}\right)\right)=\max \left\{d\left(x_{1}, x_{2}\right), d\left(y_{1}, y_{2}\right)\right\} .
$$

As we will work in a semigroup of maps without the identity element, we will consider the set $\mathbb{N}$ as the set of all positive integers.

\section{Topological Spaces of Open Sets}

Let $\mathcal{U}(X)$ denote the space of all non-empty connected open subsets of $X$, and $\mathbb{U}(X)=$ $\mathcal{U}(X) / \sim$ be the quotient space by the equivalence relation $U \sim V \Leftrightarrow \bar{U}=\bar{V} \Leftrightarrow$ $\widehat{U}=\widehat{V}$, where $\widehat{U}$ denotes the interior of $\bar{U}$. 
An open set $U \subseteq X$ is said to be geodesically $r$-regular, or simply $r$-regular, if it is a union of open geodesic balls of radius greater than or equal to $r$. Note that, as any ball of radius greater than $r$ is itself a union of balls of radius $r$, any $r$-regular open set $U$ is a union of balls of radius $r$. We will denote by $\mathcal{U}^{r}(X)$ the set of all non-empty connected $r$-regular open sets. For $r=0$ we set $\mathcal{U}^{0}(X)=\bigcup_{r>0} \mathcal{U}^{r}(X)$. Then, accordingly, the quotients of these spaces will be denoted by $\mathbb{U}^{r}(X)=\mathcal{U}^{r}(X) / \sim$, for every $r \geq 0$.

We are going to consider several topologies on the spaces $\mathcal{U}(X), \mathcal{U}^{r}(X), \mathbb{U}(X)$ and $\mathbb{U}^{r}(X)$. In the next section we will deal with topological semigroups of open maps, which will be realized as subspaces of $\mathcal{U}(X \times X)$ and $\mathbb{U}(X \times X)$. Let us now give some abstract definitions.

Definition 3.1. We say that a family $\left\{U_{\epsilon}^{*}\right\}_{\epsilon>0}$, of open connected sets, is an outer approximation of $U$, if and only if:

(1) $\bar{U}=\bigcap_{\epsilon>0} U_{\epsilon}^{*} ;$ and

(2) for all $\epsilon_{1}, \epsilon_{2}, \quad \epsilon_{1}>\epsilon_{2}>0 \Rightarrow \overline{U_{\epsilon_{2}}^{*}} \subseteq U_{\epsilon_{1}}^{*}$.

Analogously, we say that a family $\left\{U_{\epsilon}^{\circ}\right\}_{\epsilon>0}$, of open connected sets, is an inner approximation of $U$, if and only if:

(1) $U=\bigcup_{\epsilon>0} U_{\epsilon}^{\circ}$; and

(2) for all $\epsilon_{1}, \epsilon_{2}, \quad \epsilon_{1}>\epsilon_{2}>0 \Rightarrow \overline{U_{\epsilon_{1}}^{\circ}} \subseteq U_{\epsilon_{2}}^{\circ}$.

Consider any class of open sets $\mathcal{U}_{1} \subseteq \mathcal{U}(X)$.

Definition 3.2. We say that $\mathcal{U}_{1}$ is a tribe if and only if any open connected set which is a union of open sets in $\mathcal{U}_{1}$, is again in $\mathcal{U}_{1}$.

Clearly, both $\mathcal{U}(X)$ and $\mathcal{U}^{r}(X)$ are tribes, for all $r \geq 0$.

Now assume that $\mathcal{U}_{1}$ is endowed with some topology.

Definition 3.3. We say that $\mathcal{U}_{1}$ is a topological space of open sets if and only if:

(1) the topology of $\mathcal{U}_{1}$ is thinner than the Hausdorff distance topology, i.e. $\lim _{n \rightarrow \infty} U_{n}=U$ in $\mathcal{U}_{1} \Rightarrow \lim _{n \rightarrow \infty} \rho_{H}\left(U_{n}, U\right)=0$; 
(2) $\mathcal{U}_{1}$ admits outer approximations, in the sense that given $U \in \mathcal{U}_{1}$, there is an outer approximation $\left\{U_{\epsilon}^{*}\right\}_{\epsilon>0}$ of $U$ in $\mathcal{U}_{1}$ such that $\lim _{\epsilon \rightarrow 0^{+}} U_{\epsilon}^{*}=U$.

We will say that $\mathcal{U}_{1}$ admits inner approximations if and only if for every $U \in \mathcal{U}_{1}$, there is an inner approximation $\left\{U_{\epsilon}^{\circ}\right\}_{\epsilon}$ of $U$ in $\mathcal{U}_{1}$ such that $\lim _{\epsilon \rightarrow 0^{+}} U_{\epsilon}^{\circ}=U$.

It follows, from Definition 3.1(1) that the equivalence relation $\sim$ is (topologically) closed. Therefore, if $\mathcal{U}_{1}$ is a topological space of open sets then the quotient $\mathbb{U}_{1}=\mathcal{U}_{1} / \sim$ is a Hausdorff topological space. The Hausdorff distance $\rho_{H}$ is a pseudo-metric ${ }^{1}$ on $\mathcal{U}_{1}$, which induces a metric on $\mathbb{U}_{1}$. Of course the quotient topology on $\mathbb{U}_{1}$ is thinner than the metric topology of $\left(\mathbb{U}_{1}, \rho_{H}\right)$.

We now topologize the sets $\mathcal{U}(X)$ and $\mathcal{U}^{0}(X)$, turning them into topological spaces of open sets.

Consider first the following pseudo-metric $\rho$ on $\mathcal{U}(X)$. Given $U, V \in \mathcal{U}(X)$,

$$
\rho(U, V)=\max \left\{\rho_{H}(U, V), \rho_{H}\left(U^{e}, V^{e}\right)\right\} .
$$

Clearly $\rho$ satisfies $\rho_{H}(U, V) \leq \rho(U, V)$, and $\rho(U, V)=0$ if and only if $U \sim V$.

Let us say that an open set $U \subseteq X$ has regular boundary if and only if $\partial U=\partial \bar{U}$. Notice that for each open set $U \in \mathcal{U}(X), \widehat{U}$ is the unique open set with regular boundary of its equivalence class.

Proposition 3.1. Let $U \in \mathcal{U}(X)$.

(1) Defining $U_{\epsilon}^{*}=B_{\epsilon}(U)$, the family $\left\{U_{\epsilon}^{*}\right\}_{\epsilon>0}$ is an outer approximation of $U$.

(2) Defining $U_{\epsilon}^{\circ}$ to be the largest connected component of $\left\{x \in X: d\left(x, U^{c}\right)>\epsilon\right\}$, this is an open set with regular boundary and $\left\{U_{\epsilon}^{\circ}\right\}_{\epsilon>0}$ is an inner approximation of $U$.

Furthermore, for any inner approximation $\left\{U_{\epsilon}^{\circ}\right\}_{\epsilon>0}$, respectively outer approximation $\left\{U_{\epsilon}^{*}\right\}_{\epsilon>0}$, of $U$ in $\mathcal{U}(X), \lim _{\epsilon \rightarrow 0^{+}} \rho\left(U_{\epsilon}^{\circ}, U\right)=0=\lim _{\epsilon \rightarrow 0^{+}} \rho\left(U_{\epsilon}^{*}, U\right)$. In particular, with the topology associated to $\rho, \mathcal{U}(X)$ is a topological space of open sets, which admits inner approximations.

\footnotetext{
${ }^{1}$ We will use the term pseudo-metric to any function $\rho: X \times X \rightarrow[0,+\infty]$ which is symmetric and satisfies the triangle inequality. The pseudo-metric $\rho$ is called a metric if $\rho(x, y)=0$ implies $x=y$.
} 
The next proposition characterizes the topology defined by $\rho$.

Proposition 3.2. Given $U, V \in \mathcal{U}(X)$, and $\epsilon>0$,

$$
\rho(U, V) \leq \epsilon \quad \Longrightarrow \quad U_{\epsilon}^{\circ} \subseteq \widehat{V} \subseteq U_{\epsilon}^{*}
$$

Proof. First $\rho_{H}(U, V) \leq \epsilon$ implies that $\widehat{V} \subseteq \bar{V} \subseteq B_{\epsilon}(U)=U_{\epsilon}^{*}$. On the other hand $\rho_{H}\left(U^{e}, V^{e}\right) \leq \epsilon$ implies that $V^{e} \subseteq B_{\epsilon}\left(U^{e}\right) \subseteq B_{\epsilon}\left(U^{c}\right)$, which in turn implies that $U_{\epsilon}^{\circ} \subseteq \widehat{V}$.

The quotient space $\mathbb{U}(X)$ can be identified with the subspace of $\mathcal{U}(X)$ formed by all open sets which have regular boundary. With this identification, metric $\rho$ is given by

$$
\rho(U, V)=\max \left\{\rho_{H}(U, V), \rho_{H}\left(U^{c}, V^{c}\right)\right\} .
$$

Notice that $\rho$ may take infinite values. For any open set $U \in \mathcal{U}(X), \quad \rho(U, X)=$ $\rho_{H}\left(U^{c}, \varnothing\right)=+\infty$.

Proposition 3.3. With Hausdorff distance $\rho_{H}, \mathcal{U}^{r}(X)$, respectively $\mathbb{U}^{r}(X)$, is a compact pseudo-metric, respectively metric, space.

Proof. Denote by $\mathbb{K}(X)$ the space of all non-empty compact subsets of $X$. With the Hausdorff distance $\rho_{H}, \mathbb{K}(X)$ is a compact metric space (see the Blaschke selection theorem in $[\mathrm{F}])$. Therefore, it is enough to check that the correspondence $U \mapsto \bar{U}$, from $\mathcal{U}^{r}(X)$ into $\mathbb{K}(X)$ induces a one-to-one mapping from $\mathbb{U}^{r}(X)$ onto a closed subspace of $\mathbb{K}(X)$.

We consider on $\mathcal{U}^{0}(X)$ the inductive limit topology of the compact pseudo-metric spaces $\left(\mathcal{U}^{r}(X), \rho_{H}\right)$ as $r \rightarrow 0^{+}$. Then the quotient topology on $\mathbb{U}^{0}(X)$ is again the inductive limit topology of the compact metric spaces $\left(\mathbb{U}^{r}(X), \rho_{H}\right)$.

Proposition 3.4. With notation of Proposition 3.1, for any $U \in \mathcal{U}^{r}(X)$ we have $U_{\epsilon}^{*} \in \mathcal{U}^{r}(X)$ and $U_{\epsilon}^{\circ} \in \mathcal{U}^{r-\epsilon}(X)$. For each $r \geq 0, \mathcal{U}^{r}(X)$ is a topological space of open sets. For $r=0$, the inductive limit $\mathcal{U}^{0}(X)=\lim _{r \rightarrow 0^{+}} \mathcal{U}^{r}(X)$ also admits inner approximations. 
Note that $\mathcal{U}^{r}(X)$ does not admit inner approximations for $r>0$, as the open set $U=B_{r}(x) \in \mathcal{U}^{r}(X)$ fails to have inner approximations.

We will now characterize the compact topologies on $\mathcal{U}^{r}(X)$. We need the following fact.

Proposition 3.5. There is some constant $C_{r}$, depending on $r>0$ such that for all $U \in \mathcal{U}^{r}(X)$,

$$
H^{n-1}(\partial U) \leq C_{r} m(U) .
$$

In particular, every open set $U \in \mathcal{U}^{r}(X)$ is Jordan measurable, i.e. $m(\partial U)=0$.

Consider the pseudo-metrics $\rho_{H}$ and $\rho_{m}$. It is obvious that $\rho_{H}(U, V)=0 \Leftrightarrow \bar{U}=$ $\bar{V}$. Analogously, using the fact that both $U$ and $V$ are Jordan measurable open sets, one can easily checks that $\rho_{m}(U, V)=0 \Leftrightarrow \bar{U}=\bar{V}$. Therefore both $\rho_{H}$ and $\rho_{m}$ induce metrics on the quotient $\mathbb{U}^{r}(X)$.

Proposition 3.6. For each $r>0$ there is some constant $C_{r}$ such that for any pair of open sets $U, V \in \mathcal{U}^{r}(X), \quad \rho_{m}(U, V) \leq C_{r} \rho_{H}(U, V)$. The two metrics induce the same compact topology on $\mathbb{U}^{r}(X)$.

Proof. There is some constant $K_{r}$ such that $H^{n-1}(\partial U) \leq K_{r}$ for all $U \in \mathcal{U}^{r}(X)$. Letting $\epsilon=\rho_{H}(U, V), \rho_{m}(U, V)$ is bounded by the maximum volume of an $\epsilon$-neighbourhood of both $\partial U$ and $\partial V$, which is of order $\epsilon K_{r}$. It follows, using a standard abstract topology argument that the two metrics define the same Hausdorff compact topology on the quotient space.

Corollary 3.7. The real-valued function $U \mapsto m(U)$ is continuous over $\mathbb{U}^{0}(X)$.

It is enough to prove Proposition 3.5 when $X=\mathbb{R}^{n}$ and $d$ is the Euclidean distance. In general we cover $X$ with an atlas formed by a finite number of charts. Any geodesically $r$-regular open set can be decomposed as the union of a finite number of open sets in $\mathcal{U}^{r}(X)$, each of them completly covered by a single chart in the chosen atlas. Then there is some $0<r^{\prime}<r$ such that in each of these charts a geodesically $r$-regular open set is $r^{\prime}$-regular with respect to the Euclidean metric. 
Lemma 3.8. In a plane convex trapezium with both diagonals of the same length $r>0$ there is a pair of adjacent edges of length less the $r$.

Proof. There is at least an obtuse angle in the trapezium. Compare the edges incident at this vertex with the opposed diagonal.

Given a subset $A \subseteq\left\{x \in \mathbb{R}^{n}: d(x, a)=r\right\}$ define the sector

$$
S(a, A)=\{(1-t) a+t x: t \in[0,1[, x \in A\} .
$$

Lemma 3.9. Let $\Omega=B_{r}(a) \cup B_{r}(b) \subseteq \mathbb{R}^{n}$, and consider two subsets $A, B \subseteq \partial \Omega$ with $A \subseteq\left\{x \in \mathbb{R}^{n}: d(x, a)=r\right\}$ and $B \subseteq\left\{x \in \mathbb{R}^{n}: d(x, b)=r\right\}$.

$$
\text { If } A \cap B=\varnothing \text { then } S(a, A) \cap S(b, B)=\varnothing \text {. }
$$

Proof. If $S(a, A) \cap S(b, B) \neq \varnothing$ then there are points $x \in A$ and $y \in B$ such that the straight segments $[a, x]$ and $[b, y]$ intersect at some point. Consider the plane trapezium with vertices $a, y, x$ and $b$. This is a plane convex trapezium because the two diagonals $[a, x]$ and $[b, y]$ cross each other. By the previous lemma either $d(a, y)<r$ or $d(b, x)<r$ which in any case contradicts the fact that $x, y \in \partial \Omega$.

Lemma 3.10. For all $U \in \mathcal{U}^{r}\left(\mathbb{R}^{n}\right)$,

$$
H^{n-1}(\partial U) \leq \frac{\sigma_{n}}{r} m(U),
$$

where $\sigma_{n}$ is the ratio between the $(n-1)$-volume of $\left\{x \in \mathbb{R}^{n}:\|x\|=1\right\}$ and the $n$-volume of $\left\{x \in \mathbb{R}^{n}:\|x\| \leq 1\right\}$.

Proof. Denote by $H^{n-1}$ the $(n-1)$-Hausdorff measure in $\mathbb{R}^{n}$, and by $V^{n}$ the Lebesgue measure in $\mathbb{R}^{n}$. Then for any sector $S(a, A)$, with $A \subseteq\left\{x \in \mathbb{R}^{n}: d(x, a)=r\right\}$, we have

$$
\frac{H^{n-1}(A)}{V^{n}(S(a, A))}=\frac{\sigma_{n}}{r} \text {. }
$$

Claim: For any finite union $\Omega=\bigcup_{i=1}^{m} B_{r}\left(x_{i}\right)$,

$$
H^{n-1}(\partial \Omega) \leq \frac{\sigma_{n}}{r} V^{n}(\Omega) .
$$


We can cover $\partial \Omega$ with pairwise disjoint measurable sets $A_{i}$, each contained in the sphere $\left\{x \in \mathbb{R}^{n}: d\left(x, x_{i}\right)=r\right\}$. Then

$$
\begin{aligned}
H^{n-1}(\partial \Omega) & =\sum_{i=1}^{m} H^{n-1}\left(A_{i}\right) \\
& =\frac{\sigma_{n}}{r} \sum_{i=1}^{m} V^{n}\left(S\left(x_{i}, A_{i}\right)\right) \\
& \leq \frac{\sigma_{n}}{r} V^{n}(\Omega),
\end{aligned}
$$

as the sectors $S\left(x_{i}, A_{i}\right) \subseteq \Omega$ are pairwise disjoint, see Figure 1 .

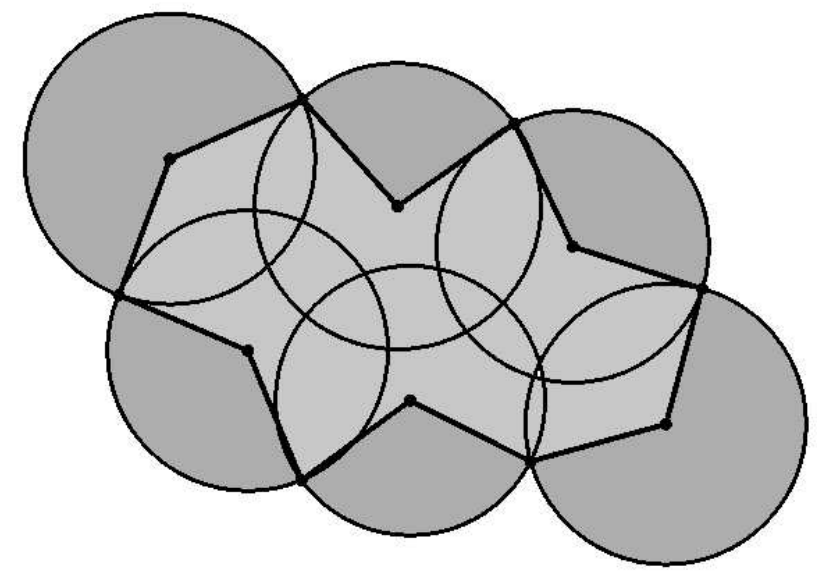

Figure 1: Pairwise disjoint sectors.

In general, if $\Omega$ is an infinite union of open balls of radius $r>0$, the proof becomes more envolved using geometric measure theory thecniques, and will be omitted here. Just to give an idea, notice that complements of $r$-regular open sets are compact sets, which are $r$-convex, in the sense that they are intersections of an arbitrary number of open ball complements $\left\{x \in \mathbb{R}^{n}: d(x, a) \geq r\right\}$. These $r$-convex sets share with convex sets some nice properties. Given any $r$-convex set $K \subseteq \mathbb{R}^{n}$ with connected complement there is $\delta_{0}(K)>0$ and a Lispchitz projection $p: B_{\delta_{0}}(K) \rightarrow K$ such that $d(x, K)=\|x-p(x)\|$, for all $x \in B_{\delta_{0}}(K)$. Furthermore $\varphi(x)=d^{2}(x, K)$ is a class $C^{1}$ function with gradient $\nabla \varphi(x)=2(x-p(x))$. These objects can be used to find Lispchitz parametrizations of the boundary $\partial \Omega$. 


\section{Topological Semigroups of Open Maps}

Let $\mathcal{S}(X)$ be the space of all point-set maps on $X$, that is, $\varphi \in \mathcal{S}$ if and only if $\varphi$ is a map from $X$ into the power set of $X$, i.e. $\varphi: X \rightarrow \mathcal{P}(X)$. For a point-set map $\varphi \in \mathcal{S}$ and a subset $A \subseteq X$ the image $\varphi(A) \in \mathcal{P}(X)$ is defined by $\varphi(A)=\cup_{x \in A} \varphi(x)$. We say that $A \subseteq X$ is $\varphi$-invariant when $\varphi(A) \subseteq A$. Analogously, we say that $A$ is fully $\varphi$-invariant if $\varphi(A)=A$. For two point-set maps $\varphi, \psi \in \mathcal{S}$ the usual composition product $\varphi \circ \psi: X \rightarrow \mathcal{P}(X)$ of $\varphi$ and $\psi$ at $x$ is defined by

$$
(\varphi \circ \psi)(x)=\varphi(\psi(x))=\cup_{y \in \psi(x)} \varphi(y) .
$$

Clearly, $\mathcal{S}(X)$ with this composition product is a semigroup.

We say that a point-set map $\varphi \in \mathcal{S}(X)$ is open when

$$
\operatorname{graph} \varphi=\{(x, y) \in X \times X: y \in \varphi(x)\}
$$

is an open subset of $X \times X$.

We define $\mathcal{O}(X)$ to be the space of all point-set maps $\varphi$ in $\mathcal{S}(X)$ that satisfy:

(1) $\varphi$ is open; and

(2) $\varphi(x)$ is non-empty and connected for every $x \in X$,

that is, $\varphi \in \mathcal{O}(X)$ if and only if $\varphi$ is an open point-set map on $X$ with connected non-empty values.

Proposition 4.1. Given $\varphi \in \mathcal{O}(X)$ and an open set $C \subseteq X$, if $C$ is connected then $\varphi(C)$ is open and connected.

Proof. Let $C \subseteq X$ be an open connected set. It is clear that $\varphi(C)$ is open, since $\varphi$ is open. Assume, by contradiction, that $Y=\varphi(C)$ is not connected. There are then non-empty, disjoint, open sets $A$ and $B$ such that $Y=A \cup B$. Clearly,

$$
\varphi^{-1}(A)=\{x \in C: \varphi(x) \cap A \neq \varnothing\} \text { and } \varphi^{-1}(B)=\{x \in C: \varphi(x) \cap B \neq \varnothing\}
$$

are non-empty open subsets of $C$ whose union is $C$. Let us show that $\varphi^{-1}(A)$ and $\varphi^{-1}(B)$ are disjoint. Again, by contradiction, we suppose there is $x \in C$ such that 
$\varphi(x) \cap A \neq \varnothing$ and $\varphi(x) \cap B \neq \varnothing$. Since $A$ and $B$ are disjoint, $\varphi(x)$ is not connected and we get a contradiction. Thus, $\varphi^{-1}(A)$ and $\varphi^{-1}(B)$ are disjoint. We conclude that $C$ is not connected. We have reached a contradiction, so $\varphi(C)$ is connected.

From the previous proposition we easily have the following.

Proposition 4.2. $\mathcal{O}(X)$ is a subsemigroup of $\mathcal{S}(X)$.

Given $\varphi \in \mathcal{O}(X)$ we define $\bar{\varphi}, \widehat{\varphi}: X \rightarrow \mathcal{P}(X)$ by setting

$$
\begin{gathered}
\operatorname{graph} \bar{\varphi}=\overline{\operatorname{graph} \varphi} \quad \text { and } \\
\operatorname{graph} \widehat{\varphi}=(\overline{\operatorname{graph} \varphi})^{\circ} .
\end{gathered}
$$

We say that $\varphi_{1}, \varphi_{2} \in \mathcal{O}(X)$ are equivalent, and write $\varphi_{1} \sim \varphi_{2}$, if and only if $\overline{\varphi_{1}}=\overline{\varphi_{2}}$, or, which is equivalent, $\widehat{\varphi_{1}}=\widehat{\varphi_{2}}$.

Proposition 4.3. For all $\varphi, \psi \in \mathcal{O}(X), \varphi \circ \psi \sim \widehat{\varphi} \circ \widehat{\psi}$.

Proof. It is enough to prove that $\widehat{\varphi} \circ \psi \sim \varphi \circ \psi \sim \varphi \circ \widehat{\psi}$. To prove the first equivalence note that $\operatorname{graph}(\varphi \circ \psi) \subseteq \operatorname{graph}(\widehat{\varphi} \circ \psi)$. Thus it is enough to see that first of these graphs is dense in the second. Take $(x, z) \in \operatorname{graph}(\widehat{\varphi} \circ \psi)$. Then there exists $y \in \psi(x)$ such that $z \in \hat{\varphi}(y)$. As $\operatorname{graph}(\varphi)$ is dense in $\operatorname{graph}(\widehat{\varphi})$, there is a sequence $\left(y_{n}, z_{n}\right) \in \operatorname{graph}(\varphi)$ converging to $(y, z)$. Because $\psi(x)$ is open, we have $y_{n} \in \psi(x)$ for all large $n$, which proves that $\left(x, z_{n}\right) \in \operatorname{graph}(\varphi \circ \psi)$ approximates $(x, z)$ as $n$ tends to infinity. Therefore $\operatorname{graph}(\varphi \circ \psi)$ is dense in $\operatorname{graph}(\widehat{\varphi} \circ \psi)$.

In a similar way, we can prove that $\operatorname{graph}(\varphi \circ \psi)$ is dense in $\operatorname{graph}(\varphi \circ \widehat{\psi})$, which implies the second equivalence.

From this proposition follows that the relation $\sim$ is compatible with composition. In particular the quotient $\mathbb{O}(X)=\mathcal{O}(X) / \sim$ inherits a semigroup structure from the usual composition. As a set, $\mathbb{O}(X)$ can be identified with the subspace of all $\varphi \in \mathcal{O}(X)$ such that $\operatorname{graph}(\varphi)$ is an open set with regular boundary. We will say that such open maps $\varphi$ have regular boundary. Notice that compositions in $\mathbb{O}(X)$ and $\mathcal{O}(X)$ are different. Given $\varphi, \psi \in \mathbb{O}(X)$, regarded as open maps with regular boundary, if $\varphi \circ \psi=\zeta$ in $\mathcal{O}(X)$ then $\varphi \circ \psi=\widehat{\zeta}$ in $\mathbb{O}(X)$.

From Definition 3.2, we have the following proposition. 
Proposition 4.4. Given any tribe of open sets $\mathcal{U}_{1} \subseteq \mathcal{U}(X)$ the set

$$
\mathcal{O}_{1}=\left\{\varphi \in \mathcal{O}(X): \forall x \in X, \varphi(x) \in \mathcal{U}_{1}\right\}
$$

is a subsemigroup of $\mathcal{O}(X)$.

Given $r>0$, we say that a map $\varphi \in \mathcal{O}(X)$ is $r$-regular if for every $x \in X$ the open set $\varphi(x)$ is $r$-regular, i.e. $\varphi(x) \in \mathcal{U}^{r}(X)$. We will denote by $\mathcal{O}^{r}(X)$ the subset of all $r$-regular open maps. For $r=0$ we set $\mathcal{O}^{0}(X)=\bigcup_{r>0} \mathcal{O}^{r}(X)$. As each $\mathcal{O}^{r}(X)$ is a tribe we have the following from the previous proposition.

Proposition 4.5. For all $r \geq 0, \mathcal{O}^{r}(X)$ is a subsemigroup of $\mathcal{O}(X)$

Of course, setting $\mathbb{O}^{r}(X)=\mathcal{O}^{r}(X) / \sim$, this is a subsemigroup of $\mathbb{O}(X)$.

We say that a point-set map is Lipschitz if and only if the map $x \mapsto \varphi(x)$ is Lipschitz with respect to the Hausdorff distance $\rho_{H}$, i.e. there is $K>0$ such that $\rho_{H}(\varphi(x), \varphi(y)) \leq K d(x, y)$ for every $y \in X$. We shall denote by $\operatorname{Lip}(\varphi)$ the greatest lower bound of all Lipschitz constants $K$ for this map. We denote by $\mathcal{O}_{\text {Lip }}(X)$ the subset of all Lipschitz point-set maps in $\mathcal{O}(X)$.

We recall the following continuity concepts. A point-set map $\varphi: X \rightarrow \mathcal{P}(X)$ with non-empty values is called lower semi-continuous, respectively upper semi-continuous, if for every $x \in X$ and $\epsilon>0$ there is a neighborhood $N_{x}$ of $x$ in $X$ such that for any $y \in N_{x}, \varphi(x) \subseteq B_{\epsilon}(\varphi(y))$, respectively $\varphi(y) \subseteq B_{\epsilon}(\varphi(x))$. It is called continuous if $\varphi: X \rightarrow \mathcal{P}(X)$ is both lower semi-continuous and upper semi-continuous. This means continuity with respect to the Hausdorff distance. We denote by $\mathcal{O}_{\text {Cont }}(X)$ the subset of all continuous point-set maps in $\mathcal{O}(X)$.

Proposition 4.6. $\mathcal{O}_{\text {Lip }}(X)$ and $\mathcal{O}_{\text {Cont }}(X)$ are both subsemigroups of $\mathcal{O}(X)$.

Proof. Let $\varphi, \psi \in \mathcal{O}_{\text {Lip }}$ with constants $K=\operatorname{Lip}(\varphi)$ and $M=\operatorname{Lip}(\psi)$. Take $x_{1}, x_{2} \in X$ with $r=d\left(x_{1}, x_{2}\right)$. It is enough to prove that $(\psi \circ \varphi)\left(x_{2}\right) \subseteq \bar{B}_{M K r}\left((\psi \circ \varphi)\left(x_{1}\right)\right)$. Then, by symmetry, exchanging the roles of $x_{1}$ and $x_{2}$ we get

$$
\rho_{H}\left((\psi \circ \varphi)\left(x_{1}\right),(\psi \circ \varphi)\left(x_{2}\right)\right) \leq M K r=M K d\left(x_{1}, x_{2}\right) .
$$


Given $z_{2} \in(\psi \circ \varphi)\left(x_{2}\right)$ there is $y_{2} \in \varphi\left(x_{2}\right)$ such that $z_{2} \in \psi\left(y_{2}\right)$. Since $\varphi$ is Lipschitz, with $\operatorname{Lip}(\varphi)=K$, we obtain $y_{2} \in \bar{B}_{K r}\left(\varphi\left(x_{1}\right)\right)$. Therefore, there exists $y_{1} \in \varphi\left(x_{1}\right)$ such that $d\left(y_{2}, y_{1}\right) \leq K r$. Because $\psi$ is also Lipschitz, with $\operatorname{Lip}(\psi)=M$, we obtain

$$
z_{2} \in \bar{B}_{M K r}\left(\psi\left(y_{1}\right)\right) \subseteq \bar{B}_{M K r}\left((\psi \circ \varphi)\left(x_{1}\right)\right)
$$

We will denote by $\mathbb{O}_{\text {Lip }}(X)$ and $\mathbb{O}_{\text {Cont }}(X)$ the corresponding subsemigroups of $\mathbb{O}(X)$. For any $r \geq 0$, define the semigroups $\mathcal{O}_{\text {Lip }}^{r}=\mathcal{O}_{\text {Lip }} \cap \mathcal{O}^{r}$ and $\mathcal{O}_{\text {Cont }}^{r}=\mathcal{O}_{\text {Cont }} \cap \mathcal{O}^{r}$. Accordingly, their quotients will be denoted by $\mathbb{O}_{\text {Lip }}^{r}$ and $\mathbb{O}_{\text {Cont }}^{r}$.

The following proposition is easily proved.

Proposition 4.7. (1) All maps $\varphi$ in $\mathcal{O}(X)$ are lower semi-continuous.

(2) For every map $\varphi \in \mathcal{O}(X), \bar{\varphi}$ is upper semi-continuous.

(3) Every map $\varphi \in \mathcal{O}_{\text {Lip }}(X)$ is continuous.

Identifying each $\varphi \in \mathcal{O}(X)$ with its graph $\operatorname{graph}(\varphi)$ we can see $\mathcal{O}(X)$, respectively $\mathbb{O}(X)$, as a subset of $\mathcal{U}(X \times X)$, respectively $\mathbb{U}(X \times X)$. We are going to consider some topologies on the semigroups $\mathcal{O}(X), \mathcal{O}_{\text {Lip }}^{r}(X), \mathbb{O}(X)$ and $\mathbb{O}_{\text {Lip }}^{r}(X)$. Let us now give some abstract definitions.

Definition 4.1. We say that a family of open point-set maps $\left\{\varphi_{\epsilon}\right\}_{\epsilon>0}$ is an outer approximation, respectively inner approximation, of $\varphi \in \mathcal{O}$ when $\left\{\text { graph } \varphi_{\epsilon}\right\}_{\epsilon>0}$ is an outer, respectively inner, approximation of graph $\varphi$.

Given open maps $\varphi, \psi: X \rightarrow \mathcal{P}(X)$, we will write $\varphi \leq \psi$ to mean that $\operatorname{graph}(\varphi) \subseteq$ $\operatorname{graph}(\psi)$, and $\varphi \prec \psi$ to say that $\overline{\operatorname{graph}(\varphi)} \subseteq \operatorname{graph}(\psi)$. Clearly, these are transitive relations compatible with compositions.

Consider any subsemigroup of open maps $\mathcal{O}_{1} \subseteq \mathcal{O}(X)$, endowed with some topo$\log y$. 
Definition 4.2. We say that $\mathcal{O}_{1}$ is a topological semigroup of open maps if and only if:

(a) $\mathcal{O}_{1}$ is a topological space of open sets in $\mathcal{U}(X \times X)$, in the sense of Definition 3.3;

(b) given $\epsilon>0$, an integer $N \in \mathbb{N}$, and non-empty open subsets $U, V \subseteq X$ such that $\overline{U \times V} \subseteq \operatorname{graph} \varphi^{N}$, there is a neighbourhood $\mathcal{N}$ of $\varphi$ in $\mathcal{O}_{1}$ such that for all $\psi \in \mathcal{N}$ and $x \in \bar{U}, \quad m\left(V \backslash \widehat{\psi^{N}}(x)\right)<\epsilon$.

If $\mathcal{O}_{1}$ is a topological semigroup of open maps, we will say that the quotient semigroup $\mathbb{O}_{1}=\mathcal{O}_{1} / \sim$ is also a topological semigroup of open maps. Alternatively, regarding elements in $\mathbb{O}_{1}$ as open maps with regular boundary, a similar definition as above can be used to characterize when is $\mathbb{O}_{1}$ a topological semigroup of open maps. Notice that condition (b) becomes even simpler because, in the quotient semigroup, $\psi^{N}$ will play the role that $\widehat{\psi^{N}}$ has in $\mathcal{O}_{1}$.

We say that a topological semigroup $\mathcal{O}_{1}$ is lower semi-continuous if and only if given inner approximations in $\mathcal{O}_{1},\left\{\varphi_{\epsilon}^{\circ}\right\}_{\epsilon>0}$ and $\left\{\psi_{\epsilon}^{\circ}\right\}_{\epsilon>0}$ of $\varphi, \psi \in \mathcal{O}_{1}$, respectively, if $\lim _{\epsilon \rightarrow 0^{+}} \varphi_{\epsilon}^{\circ}=\varphi$ and $\lim _{\epsilon \rightarrow 0^{+}} \psi_{\epsilon}^{\circ}=\psi$, then $\lim _{\epsilon \rightarrow 0^{+}} \psi_{\epsilon}^{\circ} \circ \varphi_{\epsilon}^{\circ}=\psi \circ \varphi$.

When the composition mapping is continuous we will say that $\mathcal{O}_{1}$ is continuous.

Proposition 4.8. Given a topological semigroup of open maps $\mathbb{O}_{1}$ and $N \in \mathbb{N}$, the family of mappings $\varphi \mapsto \varphi^{N}(x)$ from $\mathbb{O}_{1}$ into $\left(\mathbb{U}(X), \rho_{H}\right)$ is lower semi-equicontinuous, i.e. given $\epsilon>0$ there is a neighbourhood $\mathcal{N}$ of $\varphi$ in $\mathbb{O}_{1}$ such that for all $\psi \in \mathcal{N}$ and $x \in X, B_{\epsilon}\left(\psi^{N}(x)\right) \supseteq \varphi^{N}(x)$.

Proof. Given $\epsilon>0$ cover graph $\overline{\varphi^{N}}$ by a finite number of $\epsilon$-radius balls $B_{\epsilon}\left(x_{i}, y_{i}\right)=$ $B_{\epsilon}\left(x_{i}\right) \times B_{\epsilon}\left(y_{i}\right), \quad i=1, \cdots, m$. Then, for each $i$, choose a neighbourhood $\mathcal{N}_{i}$ of $\varphi$ in $\mathbb{O}_{1}$ such that for all $\psi \in \mathcal{N}_{i}$ and $x \in \bar{B}_{\epsilon}\left(x_{i}\right), \quad m\left(B_{\epsilon}\left(y_{i}\right) \backslash \psi^{N}(x)\right)<m\left(B_{\epsilon}\left(y_{i}\right)\right)$, which in turn implies that $\psi^{N}(x) \cap B_{\epsilon}\left(y_{i}\right) \neq \varnothing$. Given $\psi \in \cap_{i=1}^{m} \mathcal{N}_{i}, x \in X$ and $y \in \varphi^{N}(x)$, one of the points $\left(x_{i}, y_{i}\right)$ is such that $(x, y) \in B_{\epsilon}\left(x_{i}, y_{i}\right)$. Taking $z \in \psi^{N}(x) \cap B_{\epsilon}\left(y_{i}\right)$ we see that $d(y, z) \leq d\left(y, y_{i}\right)+d\left(y_{i}, z\right) \leq 2 \epsilon$, which implies $y \in B_{2 \epsilon}\left(\psi^{N}(x)\right)$. This proves that $\varphi^{N}(x) \subseteq B_{2 \epsilon}\left(\psi^{N}(x)\right)$.

Proposition 4.9. Given $\varphi \in \mathcal{O}(X)$, define $\varphi_{\epsilon}^{*}$ by

$$
\operatorname{graph}\left(\varphi_{\epsilon}^{*}\right)=B_{\epsilon}(\operatorname{graph}(\varphi)) \text {, }
$$


and define $\varphi_{\epsilon}^{\circ}$ setting $\varphi_{\epsilon}^{\circ}(x)$ to be the largest connected component of the open set

$$
\left\{y \in X: d\left((x, y), \operatorname{graph}(\varphi)^{c}\right)>\epsilon\right\} .
$$

Then $\varphi_{\epsilon}^{*} \in \mathcal{O}(X)$, and $\varphi_{\epsilon}^{\circ} \in \mathcal{O}(X)$ for all small enough $\epsilon>0$. Moreover $\left\{\varphi_{\epsilon}^{\circ}\right\}_{\epsilon>0}$ and $\left\{\varphi_{\epsilon}^{*}\right\}_{\epsilon>0}$ are respectively inner and outer approximations of $\varphi$ in $\mathcal{O}(X)$.

It should be clear by definition that compositions of inner approximations is still an inner approximation.

Lemma 4.10. Given $\varphi, \psi \in \mathcal{O}(X)$, if $\left\{\varphi_{\epsilon}^{\circ}\right\}_{\epsilon>0}$ and $\left\{\psi_{\epsilon}^{\circ}\right\}_{\epsilon>0}$ are inner approximations in $\mathcal{O}(X)$ of $\varphi$ and $\psi$, respectively, then $\left\{\psi_{\epsilon}^{\circ} \circ \varphi_{\epsilon}^{\circ}\right\}_{\epsilon>0}$ is an inner approximation of $\psi \circ \varphi$.

Given $\varphi \in \mathcal{O}(X)$ and $\epsilon>0$, we will call the inner and outer approximations defined in Proposition 4.9 the $\epsilon$-implosion and $\epsilon$-explosion, respectively.

We now topologize the semigroups $\mathcal{O}(X)$ and $\mathcal{O}_{\text {Lip }}^{0}(X)$, turning them into topological semigroups of open maps. First consider $\mathcal{O}(X)$ as a subset of $\mathcal{U}(X \times X)$ and let $\rho$ be the induced pseudo-metric, which is given by $\rho(\varphi, \psi)=\rho(\operatorname{graph}(\varphi), \operatorname{graph}(\psi))$.

The following characterization of the $\epsilon$-ball for the pseudo-metric $\rho$ follows from Proposition 3.2.

Proposition 4.11. Given $\epsilon>0$, for every $\varphi, \psi \in \mathcal{O}(X)$,

$$
\rho(\varphi, \psi)<\epsilon \quad \Longrightarrow \quad \psi_{\epsilon}^{\circ} \prec \widehat{\varphi} \prec \psi_{\epsilon}^{*} .
$$

Proposition 4.12. With the topology associated to $\rho, \mathcal{O}(X)$ is a topological semigroup of open maps, which admits inner approximations and is lower semi-continuous.

Proof. Definition 4.2(a) follows from Proposition 3.1. By Proposition 4.9, $\mathcal{O}(X)$ admits inner approximations. From Lemma 4.10 and Proposition 3.1 we see that $\mathcal{O}(X)$ is lower semi-continuous. To prove Definition 4.2(b), let $U, V \subseteq X$ be nonempty open sets such that $\overline{U \times V} \subseteq \operatorname{graph} \varphi^{N}$. Taking $\delta>0$ small enough we have 
$\bar{U} \times V \subseteq \operatorname{graph}\left(\varphi_{\delta}^{\circ}\right)^{N}$. Consider the $\delta$-neighbourhood $\mathcal{N}=B_{\delta}(\varphi)$ with respect to the pseudo-metric $\rho$. If $\psi \in \mathcal{N}$ then, by Proposition 4.11, $\varphi_{\delta}^{\circ} \prec \widehat{\psi}$, implying that

$$
\overline{U \times V} \subseteq \operatorname{graph}\left(\varphi_{\delta}^{\circ}\right)^{N} \subseteq \operatorname{graph}(\widehat{\psi})^{N} \subseteq \operatorname{graph} \widehat{\psi^{N}}
$$

Therefore, $m\left(V \backslash \widehat{\psi^{N}}(x)\right)=0$ for all $x \in \bar{U}$.

It is not difficult to see that composition is not continuous. In particular, $\mathcal{O}(X)$ is not a continuous topological semigroup of open maps. However, we do have the following.

Proposition 4.13. With the topology associated to $\rho, \mathcal{O}_{\mathrm{Cont}}(X)$ is a continuous topological semigroup of open maps.

This proposition follows easily from the next lemma.

Lemma 4.14. Given $\varphi \in \mathcal{O}_{\text {Cont }}(X)$ we have, using the notation of Proposition 4.9, $\varphi_{\epsilon}^{*} \in \mathcal{O}_{\text {Cont }}(X)$, and $\varphi_{\epsilon}^{\circ} \in \mathcal{O}_{\text {Cont }}(X)$ for every small enough $\epsilon>0$. Moreover, given $\varphi, \psi \in \mathcal{O}_{\text {Cont }}(X)$, if $\left\{\varphi_{\epsilon}^{*}\right\}_{\epsilon>0}$ and $\left\{\psi_{\epsilon}^{*}\right\}_{\epsilon>0}$ are outer approximations in $\mathcal{O}_{\text {Cont }}(X)$ of $\varphi$ and $\psi$, respectively, then $\left\{\psi_{\epsilon}^{*} \circ \varphi_{\epsilon}^{*}\right\}_{\epsilon>0}$ is an outer approximation of $\psi \circ \varphi$.

In the following we introduce a semigroup topology on $\mathcal{O}_{\text {Lip }}^{0}(X)$. Consider in $\mathcal{O}(X) \subseteq \mathcal{U}(X \times X)$ the pseudo-metrics $\rho_{H}, \rho_{m}, d_{H}$ and $d_{m}$, where the last two are defined by the following definition.

Definition 4.3.

$$
\begin{aligned}
& d_{H}(\varphi, \psi)=\sup _{x \in X} \rho_{H}(\varphi(x), \psi(x)) \quad \text { and } \\
& d_{m}(\varphi, \psi)=\sup _{x \in X} \rho_{m}(\varphi(x), \psi(x)) .
\end{aligned}
$$

Given $r>0$ and $K<+\infty$, define

$$
\mathcal{O}_{K}^{r}=\left\{\varphi \in \mathcal{O}^{r}(X): \operatorname{Lip}(\varphi) \leq K\right\}
$$

Proposition 4.15. All four metrics $\rho_{H}, \rho_{m}, d_{H}$ and $d_{m}$ induce the same compact topology on $\mathcal{O}_{K}^{r}$. 
Proof. In general one has $\rho_{H}(\varphi, \psi) \leq d_{H}(\varphi, \psi)$ over $\mathcal{O}(X)$. If $\varphi$ and $\psi$ are Lipschitz with Lipschitz constant $K$, then we can also prove that $d_{H}(\varphi, \psi) \leq K \rho_{H}(\varphi, \psi)$. Therefore, $\rho_{H}$ and $d_{H}$ are Lipschitz equivalent over $\mathcal{O}_{K}^{r}$.

It is clear that

$$
\rho_{m}(\varphi, \psi)=\int_{X} \rho_{m}(\varphi(x), \psi(x)) d m(x) \leq d_{m}(\varphi, \psi)
$$

Using Proposition 3.6, we see that $d_{m} \leq C_{r} d_{H}$ over $\mathcal{O}^{r}$ for some constant $C_{r}$ depending on $r>0$.

It is now enough to prove the compacity of $\mathcal{O}_{K}^{r}$ with respect to $d_{H}$. This follows by an argument as in Ascoli-Arzelá's theorem. $\mathcal{O}_{K}^{r}$ can be seen as a subset of the compact product space $\mathcal{U}^{r}(X)^{X}$. Of course $\mathcal{O}_{K}^{r}$ is closed and, therefore, compact for the product topology. The set $\mathcal{O}_{K}^{r}$ is equicontinuous because all maps in it are Lipschitz with the same Lipschitz constant. Thus the product topology (pointwise convergence) coincides with the topology of $d_{H}$ (uniform convergence), which proves the compacity of $\mathcal{O}_{K}^{r}$ with respect to $d_{H}$.

We will consider on $\mathcal{O}_{\text {Lip }}^{0}=\bigcup_{r>0, K<\infty} \mathcal{O}_{K}^{r}$, respectively on $\mathcal{O}_{\text {Lip }}^{r}=\bigcup_{K<\infty} \mathcal{O}_{K}^{r} \quad$ if $r>0$, the inductive limit topology of the compact subspaces $\mathcal{O}_{K}^{r}$.

Proposition 4.16. With the inductive limit topology above, for each $r \geq 0, \mathcal{O}_{\text {Lip }}^{r}$ is a continuous topological semigroup of open maps.

Proof. Item (a) of Definition 4.2 is clear. The continuity of composition follows from Proposition 4.17 bellow. To see item (b) of Definition 4.2, take $\epsilon>0$ and let $U, V \subseteq X$ be non-empty open sets such that $\overline{U \times V} \subseteq \operatorname{graph} \varphi^{N}$. Consider the neighbourhood $\mathcal{N}=\left\{\psi: d_{m}\left(\psi^{N}, \varphi^{N}\right)<\epsilon\right\}$ of $\varphi$ in $\mathcal{O}_{\text {Lip }}^{r}$. Then if $\psi \in \mathcal{N}$, for all $x \in \bar{U}$

$$
m\left(V \backslash \widehat{\psi^{N}}(x)\right) \leq m\left(V \backslash \psi^{N}(x)\right) \leq m\left(\varphi^{N}(x) \backslash \psi^{N}(x)\right) \leq d_{m}\left(\psi^{N}, \varphi^{N}\right)<\epsilon
$$

Proposition 4.17. The composition map $(\varphi, \psi) \mapsto \varphi \circ \psi$ from $\mathcal{O}_{K}^{r} \times \mathcal{O}_{K}^{r}$ into $\mathcal{O}_{K^{2}}^{r}$ is continuous with respect to the compact topologies above. 
Proof. Using the lemma bellow we see that

$$
\begin{aligned}
\rho_{H}\left(\varphi_{1}\left(\psi_{1}(x)\right), \varphi_{2}\left(\psi_{2}(x)\right)\right) & \leq \rho_{H}\left(\varphi_{1}\left(\psi_{1}(x)\right), \varphi_{1}\left(\psi_{2}(x)\right)\right)+\rho_{H}\left(\varphi_{1}\left(\psi_{2}(x)\right), \varphi_{2}\left(\psi_{2}(x)\right)\right) \\
& \leq K \rho_{H}\left(\psi_{1}(x), \psi_{2}(x)\right)+d_{H}\left(\varphi_{1}, \varphi_{2}\right) \\
& \leq K d_{H}\left(\psi_{1}, \psi_{2}\right)+d_{H}\left(\varphi_{1}, \varphi_{2}\right) .
\end{aligned}
$$

Therefore

$$
d_{H}\left(\varphi_{1} \circ \psi_{1}, \varphi_{2} \circ \psi_{2}\right) \leq K d_{H}\left(\psi_{1}, \psi_{2}\right)+d_{H}\left(\varphi_{1}, \varphi_{2}\right),
$$

which proves continuity of the composition.

Lemma 4.18. For all open sets $U, V \subseteq X$, and all maps $\varphi, \psi \in \mathcal{O}(X)$ :

(1) $\rho_{H}(\varphi(U), \varphi(V)) \leq \operatorname{Lip}(\varphi) \rho_{H}(U, V)$;

(2) $\rho_{H}(\varphi(U), \psi(U)) \leq d_{H}(\varphi, \psi)$.

\section{Dynamics of Open Maps}

Given $\varphi \in \mathcal{O}(X)$, any sequence $x_{0}, x_{1}, \cdots, x_{n} \in X$ such that $x_{i} \in \varphi\left(x_{i-1}\right)$ for $i=$ $1, \cdots, n$ will be called an orbit of $\varphi$, and we will say that $x_{n}$ is an iterate of the state $x_{0}$. Given $x, y \in X$ let us write $x \rightsquigarrow_{\varphi} y$ when there exists some $n \in \mathbb{N}$ such that $y \in \varphi^{n}(x)$. The relation $\rightsquigarrow_{\varphi}$ is transitive. Moreover, $\rightsquigarrow_{\varphi}$ is an open relation, since $\varphi$ is an open point-set map. Given $A, B \subseteq X$ we will write $A \rightsquigarrow_{\varphi} B$ when $x \rightsquigarrow_{\varphi} y$ for some $x \in A$ and some $y \in B$. We define the recurrent set of $\varphi$, and denote it by $\Omega(\varphi)$, as the set of all states $x \in X$ such that $x$ is an iterate of $x$, that is,

$$
\Omega(\varphi)=\left\{x \in X: x \rightsquigarrow_{\varphi} x\right\} .
$$

Clearly, $\Omega(\varphi)$ is an open set. On $\Omega(\varphi)$ the relation $\rightsquigarrow_{\varphi}$ is reflexive and transitive and thus is a preorder. We call the elements of $\Omega(\varphi)$ recurrent states. The recurrent set $\Omega(\varphi)$ can be decomposed into classes defined by the following equivalence relation. Given $x, y \in X$ we will write $x \rightsquigarrow_{\varphi} y$ when $x=y$, or then $x \rightsquigarrow_{\varphi} y$ and $y \rightsquigarrow_{\varphi} x$. Since $\rightsquigarrow_{\varphi}$ is a preorder on $\Omega(\varphi), m_{\varphi}$ is an equivalence relation. The equivalence classes of 
recurrent states will be called $\Omega$-classes. Every $\Omega$-class is open, since $\varkappa_{\varphi}$ is an open relation. We will denote by $\Lambda^{\Omega}(\varphi)$ the set of all $\Omega$-classes of $\varphi$.

The relation $\rightsquigarrow_{\varphi}$ imposes a partial ordering on $\Lambda^{\Omega}(\varphi)$. Indeed the relation $\rightsquigarrow_{\varphi}$ is a preorder on $\Lambda^{\Omega}(\varphi)$ and in addition for $A, B \in \Lambda^{\Omega}(\varphi), A \rightsquigarrow \varphi B$ and $B \rightsquigarrow \varphi A$ together imply $A=B$. The maximal elements in $\Lambda^{\Omega}(\varphi)$ under $\rightsquigarrow \varphi$ will be called $\Omega$-final classes. In other words $D \in \Lambda^{\Omega}(\varphi)$ is an $\Omega$-final class if for all $C \in \Lambda^{\Omega}(\varphi)$

$$
D \rightsquigarrow_{\varphi} C \Rightarrow C=D .
$$

We will denote by $\Lambda_{\text {final }}^{\Omega}(\varphi)$ the set of all $\Omega$-final components of $\varphi$.

A recurrent state $x$ will be called a final recurrent state if every iterate of $x$ still has some iterate which comes back to $x$, that is,

$$
\Omega_{\text {final }}(\varphi)=\left\{x \in \Omega(\varphi): \text { for every } y \in X, x \rightsquigarrow_{\varphi} y \Rightarrow y \rightsquigarrow_{\varphi} x\right\} \text {, }
$$

where we denote by $\Omega_{\text {final }}(\varphi)$ the set of all final recurrent states. We will call $\Omega_{\text {final }}(\varphi)$ the final recurrent set. Indeed this is the set where all the dynamics will eventually end up. It should be regarded as the union of all "attractors" of $\varphi$. Clearly, final recurrent states and $\Omega$-final classes are related in the following obvious way.

Proposition 5.1. $\Omega_{\text {final }}(\varphi)$ is the union of all $\Omega$-final classes in $C \in \Lambda_{\text {final }}^{\Omega}(\varphi)$, each $\Omega$-final class being fully $\varphi$-invariant, i.e. $\varphi(C)=C$.

For a subset $C \subseteq X$ we define the following open sets:

$$
\begin{aligned}
& W_{\Omega}^{\mathrm{s}}(C, \varphi)=W_{\Omega}^{\mathrm{s}}(C)=\left\{z \in X: z \rightsquigarrow_{\varphi} C\right\}, \text { and } \\
& W_{\Omega}^{\mathrm{u}}(C, \varphi)=W_{\Omega}^{\mathrm{u}}(C)=\left\{z \in X: C \rightsquigarrow_{\varphi} z\right\},
\end{aligned}
$$

which we will call, respectively, the $\Omega$-stable and $\Omega$-unstable sets of $C$.

The following proposition implies a uniform spread on images of open maps. It follows by compacity of $X$ using a standard type of argument.

Proposition 5.2. Given $\varphi \in \mathcal{O}(X)$ there is a map $F: X \rightarrow X$ and $\xi_{0}>0$ such that

$$
B_{\xi_{0}}(\operatorname{graph}(F)) \subseteq \operatorname{graph}(\varphi) .
$$


Proposition 5.3. The partial ordered set $\Lambda_{\text {final }}^{\Omega}(\varphi)$ is finite, and every state $x \in X$ has some iterate in $\Omega_{\mathrm{final}}(\varphi)$, i.e. $X=W_{\Omega}^{\mathrm{s}}\left(\Omega_{\mathrm{final}}(\varphi)\right)$.

In other words, $\Omega_{\text {final }}(\varphi)$ splits into a finite number of attractors, attracting everything.

Proof. By Proposition 5.2 there is some $\xi_{0}>0$ such that any $\Omega$-final class, being fully $\varphi$-invariant, contains a ball of radius $\xi_{0}>0$. Therefore the volumes of $\Omega$-final classes are uniformly bounded from zero. Since $X$ is compact it follows that $\Lambda_{\text {final }}^{\Omega}(\varphi)$ must be finite. The second statement of this proposition is a consequence of the following two lemmas.

Lemma 5.4. $X=W_{\Omega}^{\mathrm{s}}(\Omega(\varphi))$.

Proof. Let $F$ and $\xi_{0}>0$ be as given by Proposition 5.2. Take $x_{0} \in X$ and define $x_{n}=F^{n}\left(x_{0}\right)$ for every $n \in \mathbb{N}$. Let $z$ be a sublimit of $x_{n}$. If $d\left(x_{n}, z\right)<\xi_{0}$ and $d\left(x_{n+p}, z\right)<\xi_{0}$, then $x_{n+1} \in \varphi(z)$ and $z \in \varphi\left(x_{n+p-1}\right)$. So $z \rightsquigarrow_{\varphi} z$ and thus $z \in \Omega(\varphi)$. Since $x_{0} \rightsquigarrow_{\varphi} x_{n+p-1}$ and $x_{n+p-1} \rightsquigarrow_{\varphi} z$, we have $x_{0} \rightsquigarrow_{\varphi} z$.

Lemma 5.5. For each $\Omega$-class $D \in \Lambda^{\Omega}(\varphi)$ there is some $\Omega$-final class $D_{0} \in \Lambda_{\text {final }}^{\Omega}(\varphi)$ such that $D \rightsquigarrow_{\varphi} D_{0}$.

Proof. By Zorn's lemma it is enough to prove that each forward chain $\mathcal{D} \subseteq \Lambda^{\Omega}(\varphi)$ has some upper bound $C \in \Lambda^{\Omega}(\varphi)$, i.e. for all $D \in \mathcal{D}, D \rightsquigarrow_{\varphi} C$. A forward chain $\mathcal{D}$ in $\left(\Lambda^{\Omega}(\varphi), \rightsquigarrow_{\varphi}\right)$ is a subset $\mathcal{D} \subseteq \Lambda^{\Omega}(\varphi)$ which is well ordered ${ }^{2}$ by $\rightsquigarrow_{\varphi}$. Assume there is no $z \in X$ such that for all $D \in \mathcal{D}, \quad D \rightsquigarrow_{\varphi} z$. Define for each $D \in \mathcal{D}$,

$$
S_{D}=\bigcap_{C \in \mathcal{D}, C \backsim \varphi D} W_{\Omega}^{\mathrm{u}}(C) \backslash W_{\Omega}^{\mathrm{u}}(D) .
$$

Then the family $\left\{S_{D}\right\}_{D \in \mathcal{D}}$ is formed of pairwise disjoint sets, and so we have $\sum_{D \in \mathcal{D}} m\left(S_{D}\right)<$ $\infty$. Thus, there is some $D \in \mathcal{D}$ such that

$$
m\left(\bigcup_{C \leftrightarrow \varphi_{\varphi} D} S_{C}\right)=\sum_{C \not \leftrightarrow \varphi D} m\left(S_{C}\right)<c_{0},
$$

\footnotetext{
${ }^{2} \mathrm{~A}$ well ordered set is a totally ordered set such that every non-empty subset has a first element.
} 
where $c_{0}$ is the $m$-volume of a ball of radius $\xi_{0}$, for $\xi_{0}$ as in Proposition 5.2. By the above assumption, we have $\bigcup_{C \ngtr \hookrightarrow \varphi} S_{C}=\bigcap_{C \rightsquigarrow \varphi D} W_{\Omega}^{\mathrm{u}}(C)$. Clearly this set is $\varphi$-invariant which contradicts the spread property of $\varphi$ given by Proposition 5.2. Therefore our assumption must be false and so there is some $z \in X$ such that for all $D \in \mathcal{D}, D \rightsquigarrow \varphi z$. By Proposition 5.4 there is some class $C \in \Lambda^{\Omega}(\varphi)$ such that $z \rightsquigarrow_{\varphi} C$. Thus, for all $D \in \mathcal{D}, D \rightsquigarrow_{\varphi} C$.

A connected component of $\Omega(\varphi)$, respectively $\Omega_{\text {final }}(\varphi)$, will be called an $\Omega$-component, respectively an $\Omega$-final component, of $\varphi$. We denote respectively by $\Sigma^{\Omega}(\varphi)$ and $\Sigma_{\text {final }}^{\Omega}(\varphi)$ the sets of all $\Omega$-components, and of all $\Omega$-final components, of $\varphi$.

Theorem 5.1. The set of $\Omega$-final components $\Sigma_{\text {final }}^{\Omega}(\varphi)$ is finite. For every component $C \in \Sigma_{\text {final }}^{\Omega}(\varphi), \varphi(C) \in \Sigma_{\text {final }}^{\Omega}(\varphi)$. The mapping $\pi_{\varphi}: \Sigma_{\text {final }}^{\Omega}(\varphi) \rightarrow \Sigma_{\text {final }}^{\Omega}(\varphi)$, defined by $\pi_{\varphi}(C)=\varphi(C)$, is a bijection, which permutes ciclically the components of each $\Omega$-final class.

Proof. By Proposition 5.1, every $\Omega$-final class $C$ satisfies $\varphi(C)=C$. Thus, for every component $C \in \Sigma_{\text {final }}^{\Omega}(\varphi)$, since $\varphi(C)$ is also connected, $\varphi(C)$ must be another final component in $\Sigma_{\text {final }}^{\Omega}(\varphi)$. It follows by Proposition 5.2 , that every $\Omega$-final component must contain some ball of minimum radius $\xi_{0}$. Therefore $\Sigma_{\text {final }}^{\Omega}(\varphi)$ is finite. Because each component $C \in \Sigma_{\text {final }}^{\Omega}(\varphi)$ is formed by recurrent states, there is some integer $n \in \mathbb{N}$ such that $\varphi^{n}(C)=C$. By definition of an $\Omega$-class it follows that $\cup_{i=0}^{n-1} \varphi^{i}(C)$ is precisely the class of $C$.

Given an $\Omega$-final class $C$, we will call period of $C$ to the number of its connected components. Given a component $C_{0}$ of $C$, we will call period of $C_{0}$ to the period of its class $C$.

Next we introduce chain recurrence and relate it to the former recurrence concept. Given $\varphi \in \mathcal{O}(X)$ and $\epsilon>0, \varphi_{\epsilon}^{*}$ will denote the $\epsilon$-explosion of $\varphi$ as defined in the previous section. Any sequence $x_{0}, x_{1}, \cdots, x_{n} \in X$ such that $x_{i} \in \varphi_{\epsilon}^{*}\left(x_{i-1}\right)$ for $i=1, \cdots, n$ will be called an $\epsilon$-pseudo-orbit of $\varphi$, and we will say that $x_{n}$ is an $\epsilon$-pseudo-iterate of the state $x_{0}$. If $y$ is an $\epsilon$-pseudo-iterate of $x$ for every $\epsilon>0, y$ is said to be a pseudo-iterate of $x$. Given $x, y \in X$ let us write $x \rightarrow_{\varphi} y$ when $x \rightsquigarrow_{\varphi_{\epsilon}^{*}} y$ for every $\epsilon>0$. Clearly the relation $\rightarrow_{\varphi}$ is a transitive, closed relation. Given $A, B \subseteq X$ we will write $A \rightarrow_{\varphi} B$ when $x \rightarrow_{\varphi} y$ for some $x \in A$ and some $y \in B$. 
We define the chain recurrent set of $\varphi$, and denote it by $R(\varphi)$, as the set of all states $x \in X$ such that $x$ is a pseudo-iterate of $x$, that is,

$$
R(\varphi)=\left\{x \in X: x \rightarrow_{\varphi} x\right\} .
$$

The set $R(\varphi)=\bigcap_{\epsilon>0} \overline{\Omega\left(\varphi_{\epsilon}^{*}\right)}$ is a closed set in $X$. The closed relation $\rightarrow_{\varphi}$ is a preorder on $R(\varphi)$. We call the elements of $R(\varphi)$ chain recurrent states.

The recurrent set $R(\varphi)$ can be decomposed into classes defined by the following equivalence relation. Given $x, y \in X$ we will write $x \leftrightharpoons_{\varphi} y$ when $x=y$, or then $x \rightarrow_{\varphi} y$ and $y \rightarrow_{\varphi} x$. As $\rightarrow_{\varphi}$ is a preorder on $R(\varphi), \leftrightharpoons_{\varphi}$ is an equivalence relation. The equivalence classes of recurrent states will be called $R$-classes. Every $R$-class is closed, since $\leftrightharpoons \varphi$ is a closed relation. We will denote by $\Lambda^{R}(\varphi)$ the set of all $R$-classes of $\varphi$.

The relation $\rightarrow_{\varphi}$ imposes a partial ordering on $\Lambda^{R}(\varphi)$. Indeed the relation $\rightarrow_{\varphi}$ is a preorder on $\Lambda^{R}(\varphi)$ and in addition for $A, B \in \Lambda^{R}(\varphi)$, if $A \rightarrow_{\varphi} B$ and $B \rightarrow_{\varphi} A$ then $A=B$. The maximal elements in $\Lambda^{R}(\varphi)$ under $\rightarrow_{\varphi}$ will be called $R$-final classes. In other words $D \in \Lambda^{R}(\varphi)$ is an $R$-final class if for all $C \in \Lambda^{R}(\varphi)$,

$$
D \rightarrow_{\varphi} C \Rightarrow C=D \text {. }
$$

We will denote by $\Lambda_{\text {final }}^{R}(\varphi)$ the set of all $R$-final components of $\varphi$.

A chain recurrent state $x$ will be called a final chain recurrent state if every pseudoiterate of $x$ still has some pseudo-iterate which comes back to $x$. We will call the final chain recurrent set the set, denoted by $R_{\text {final }}(\varphi)$, of all final chain recurrent states, that is,

$$
R_{\text {final }}(\varphi)=\left\{x \in R(\varphi): \text { for every } y \in X, x \rightarrow_{\varphi} y \Rightarrow y \rightarrow_{\varphi} x\right\} .
$$

Clearly, final recurrent states and $R$-final classes are related in the following obvious way.

Proposition 5.6. $R_{\text {final }}(\varphi)$ is the union of all $R$-final classes $C \in \Lambda_{\text {final }}^{R}(\varphi)$, and each such class is fully $\bar{\varphi}$-invariant, i.e. $\bar{\varphi}(C)=C$.

Proof. Given $C \in \Lambda_{\text {final }}^{R}(\varphi)$, clearly $\bar{\varphi}(C) \subseteq C$. Now, let $y \in C$, and assume, by contradiction, that $y \notin \bar{\varphi}(C)$. Then

$$
C \times\{y\} \cap \operatorname{graph} \bar{\varphi}=\varnothing .
$$


Take $x_{0} \in C$. For every $\epsilon>0, C_{\epsilon}=\bigcup_{n=1}^{\infty}\left(\varphi_{\epsilon}^{*}\right)^{n}\left(x_{0}\right)$ is the $\Omega$-class of $\varphi_{\epsilon}^{*}$ containing $x_{0}$. Of course $C=\bigcap_{\epsilon>0} C_{\epsilon}$ and $\left\{C_{\epsilon}\right\}_{\epsilon>0}$ is an outer approximation of $C$. By compactness of $C \times\{y\}$ and $\operatorname{graph} \bar{\varphi}$ there is some small enough $\epsilon>0$ such that

$$
C_{\epsilon} \times\{y\} \cap \operatorname{graph} \varphi_{\epsilon}^{*}=\varnothing
$$

which implies that $y \notin \varphi_{\epsilon}^{*}\left(C_{\epsilon}\right)=C_{\epsilon}$. But this is impossible because $y \in C \subseteq C_{\epsilon}$.

Proposition 5.7. Every $R$-final class $A \in \Lambda_{\text {final }}^{R}(\varphi)$ contains some $\Omega$-final class $C \in \Lambda_{\text {final }}^{\Omega}(\varphi), \quad A \supseteq C$.

Proof. Given $A \in \Lambda_{\text {final }}^{R}(\varphi)$, by Proposition 5.3 there is some class $C \in \Lambda_{\text {final }}^{\Omega}(\varphi)$ such that $A \rightsquigarrow_{\varphi} C$, which in turn implies $A \rightarrow_{\varphi} C$. Thus $A \supseteq C$, since $A$ is an $R$-final class.

A connected component of $R(\varphi)$, respectively $R_{\text {final }}(\varphi)$, will be called an $R$-component, respectively an $R$-final component, of $\varphi$. We denote respectively by $\Sigma^{R}(\varphi)$ and $\Sigma_{\text {final }}^{R}(\varphi)$ the sets of all $R$-components, and of all $R$-final components, of $\varphi$.

Theorem 5.2. The set of $R$-final components $\Sigma_{\text {final }}^{R}(\varphi)$ is finite. For every component $A \in \Sigma_{\text {final }}^{R}(\varphi), \bar{\varphi}(A) \in \Sigma_{\text {final }}^{\Omega}(\varphi)$. The mapping $\bar{\pi}_{\varphi}: \Sigma_{\text {final }}^{R}(\varphi) \rightarrow \Sigma_{\text {final }}^{R}(\varphi)$, defined by $\bar{\pi}_{\varphi}(A)=\bar{\varphi}(A)$, is a bijection, which permutes ciclically the components of each $R$-final class.

Proof. Given any component $A \in \Sigma_{\text {final }}^{R}(\varphi)$, as $\bar{\varphi}(A)$ is connected, it is completly contained inside a single component $A^{\prime} \in \Sigma_{\text {final }}^{R}(\varphi)$. Let us define $\bar{\pi}_{\varphi}: \Sigma_{\text {final }}^{R}(\varphi) \rightarrow$ $\Sigma_{\text {final }}^{R}(\varphi)$, setting $\bar{\pi}_{\varphi}(A)=A^{\prime}$. By Proposition 5.1, $\bar{\pi}_{\varphi}$ is surjective. Therefore each component of $\Sigma_{\text {final }}^{R}(\varphi)$ contains some image $\bar{\varphi}(x)$ and, by Proposition 5.2, must have a minimum volume. By compacity of $X$ the set of all components $\Sigma_{\text {final }}^{R}(\varphi)$ is finite. Thus, $\bar{\pi}_{\varphi}$ must be one-to-one. Again by the same Proposition 5.1, $\bar{\pi}_{\varphi}$ permutes the components of each $R$-final class. Finally, this implies that $\bar{\varphi}(A)$ is a whole component, for each $A \in \Sigma_{\text {final }}^{R}(\varphi)$.

We will call period of an $R$-final class $A$ to the number of its connected components. Given a component $A_{0}$ of $A$, we will call period of $A_{0}$ to the period of its class $A$. 
For a subset $C \subseteq X$ we define the following closed sets:

$$
\begin{aligned}
& W_{R}^{\mathrm{s}}(C, \varphi)=W_{R}^{\mathrm{s}}(C)=\left\{z \in X: z \rightarrow_{\varphi} C\right\} \text { and } \\
& W_{R}^{\mathrm{u}}(C, \varphi)=W_{R}^{\mathrm{u}}(C)=\left\{z \in X: C \rightarrow_{\varphi} z\right\},
\end{aligned}
$$

which we will call, respectively, the $R$-stable and $R$-unstable sets of $C$. The following proposition shows that final classes behave as 'attractors' do.

Proposition 5.8. Given a final class $C \in \Lambda_{\text {final }}^{R}(\varphi)$ and a $\varphi$-invariant open set $U \subseteq X$ such that $\bar{U} \cap R(\varphi)=C, \bigcap_{n=1}^{\infty} \varphi^{n}(U) \subseteq C$.

The proposition is corollary of the following lemma.

Lemma 5.9. Given a $\varphi$-invariant open set $U \subseteq X$,

$$
\bigcap_{n=1}^{\infty} \varphi^{n}(U) \subseteq W_{R}^{\mathrm{u}}(\bar{U} \cap R(\varphi)) .
$$

Proof. Given $x \in \cap_{n=1}^{\infty} \varphi^{n}(U)$, pick, for each $n \in \mathbb{N}, z_{n} \in U$ such that $x \in \varphi^{n}\left(z_{n}\right)$. Taking $z$ to be a sublimit of $\left\{z_{n}\right\}$, we have $z \rightarrow_{\varphi} x$. Consider the compact set $\Gamma=$ $W_{R}^{\mathrm{u}}(z) \cap W_{R}^{\mathrm{s}}(x) \subseteq \bar{U}$. This set is weakly invariant in the sense that, for each $y \in \Gamma$, $\bar{\varphi}(y) \cap \Gamma \neq \varnothing$. Therefore we can define recursively a sequence $\left\{y_{n}\right\} \subseteq \Gamma$, setting $y_{0}=z$, and picking for each $n \in \mathbb{N}, y_{n+1} \in \bar{\varphi}\left(y_{n}\right) \cap \Gamma$. Let $y$ be a sublimit of $\left\{y_{n}\right\}$. Then $y \in \Gamma \cap R(\varphi) \subseteq \bar{U} \cap R(\varphi)$, which proves that $x \in W_{R}^{\mathrm{u}}(\bar{U} \cap R(\varphi))$.

Let $\varphi, \psi \in \mathcal{O}(X)$. We say that $\varphi$ is combinatorially equivalent to $\psi$, and write $\varphi \bowtie \psi$, if and only if the permutations $\pi_{\varphi}$ and $\pi_{\psi}$ are conjugated, that is, there is a bijective map $h: \Sigma_{\text {final }}^{\Omega}(\varphi) \rightarrow \Sigma_{\text {final }}^{\Omega}(\psi)$ such that the following diagram comutes:

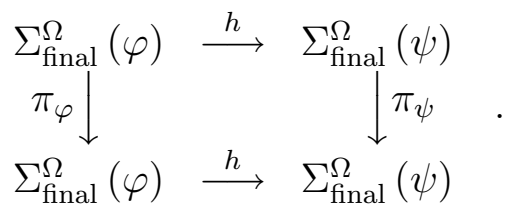

Given a topological subsemigroup $\mathcal{O}_{1} \subseteq \mathcal{O}(X)$, we say that $\varphi \in \mathcal{O}(X)$ is combinatorially stable in $\mathcal{O}_{1}$ if and only if there is a neighbourhood $\mathcal{U}$ of $\varphi$ in $\mathcal{O}_{1}$ such that all $\psi \in \mathcal{U}$ are combinatorially equivalent to $\varphi$. 
Given $\varphi, \psi \in \mathcal{O}(X)$ we define the following multi-valued mappings.

$$
\begin{aligned}
& \text { If } \varphi \leq \psi \text { let } \\
& \qquad \tau_{\varphi, \psi}: \Lambda_{\text {final }}^{\Omega}(\psi) \rightarrow \mathcal{P}\left(\Lambda_{\text {final }}^{\Omega}(\varphi)\right) \quad \text { and } \quad \theta_{\varphi, \psi}: \Sigma_{\text {final }}^{\Omega}(\psi) \rightarrow \mathcal{P}\left(\Sigma_{\text {final }}^{\Omega}(\varphi)\right)
\end{aligned}
$$

be the maps that to each $\Omega$-final class, resp. $\Omega$-final component, $C$ of $\psi$ associate the set of $\Omega$-final classes, resp. $\Omega$-final components, $C^{\prime}$ of $\varphi$ contained in $C$.

$$
\begin{aligned}
& \text { If } \varphi \prec \psi \text { let } \\
& \qquad \tau_{\varphi, \psi}^{*}: \Lambda_{\text {final }}^{\Omega}(\psi) \rightarrow \mathcal{P}\left(\Lambda_{\text {final }}^{R}(\varphi)\right) \quad \text { and } \quad \theta_{\varphi, \psi}^{*}: \Sigma_{\text {final }}^{\Omega}(\psi) \rightarrow \mathcal{P}\left(\Sigma_{\text {final }}^{R}(\varphi)\right)
\end{aligned}
$$

be the maps that to each $\Omega$-final class, resp. $\Omega$-final component, $C$ of $\psi$ associate the set of $R$-final classes, resp. $R$-final components, $A$ of $\varphi$ contained in $C$.

In general, for any $\varphi$ let

$$
\tilde{\tau}_{\varphi}: \Lambda_{\text {final }}^{R}(\varphi) \rightarrow \mathcal{P}\left(\Lambda_{\text {final }}^{\Omega}(\varphi)\right) \quad \text { and } \quad \tilde{\theta}_{\varphi}: \Sigma_{\text {final }}^{R}(\varphi) \rightarrow \mathcal{P}\left(\Sigma_{\text {final }}^{\Omega}(\varphi)\right)
$$

be the maps that to each $R$-final class, resp. $R$-final component, $A$ of $\varphi$ associate the set of $\Omega$-final classes, resp. $\Omega$-final components, $C$ of $\varphi$ contained in $A$.

In the rest of this section we aim to establish the genericity of combinatorial stability, which will follow from the key Propositions 5.16 and 5.17, where a kind of upper and lower stability of combinatorics is proved to hold for any open map. The next three propositions relate the behaviour of multi-valued mappings $\theta_{\varphi, \psi}, \theta_{\varphi, \psi}^{*}, \tilde{\theta}_{\varphi}, \tau_{\varphi, \psi}, \tau_{\varphi, \psi}^{*}$ and $\tilde{\tau}_{\varphi}$, with the order and combinatorics of open maps in $\mathcal{O}(X)$. They are both straightforward consequences of the definitions.

Proposition 5.10. Let $\varphi_{1}, \varphi_{2}, \varphi_{3} \in \mathcal{O}(X)$.

If $\varphi_{1} \prec \varphi_{2} \quad$ then $\quad \theta_{\varphi_{1}, \varphi_{2}}=\tilde{\theta}_{\varphi_{1}} \circ \theta_{\varphi_{1}, \varphi_{2}}^{*} \quad$ and $\quad \tau_{\varphi_{1}, \varphi_{2}}=\tilde{\tau}_{\varphi_{1}} \circ \tau_{\varphi_{1}, \varphi_{2}}^{*}$.

If $\varphi_{1} \leq \varphi_{2} \leq \varphi_{3}$ then $\theta_{\varphi_{1}, \varphi_{3}}=\theta_{\varphi_{1}, \varphi_{2}} \circ \theta_{\varphi_{2}, \varphi_{3}}$ and $\quad \tau_{\varphi_{1}, \varphi_{3}}=\tau_{\varphi_{1}, \varphi_{2}} \circ \tau_{\varphi_{2}, \varphi_{3}}$.

Proposition 5.11. Let $\Theta: \Xi_{1} \rightarrow \mathcal{P}\left(\Xi_{2}\right)$ stand for any of the multi-valued mappings, $\theta_{\varphi, \psi}, \theta_{\varphi, \psi}^{*}, \tilde{\theta}_{\varphi}, \tau_{\varphi, \psi}, \tau_{\varphi, \psi}^{*}$ or $\tilde{\tau}_{\varphi}$. Then for every $C \in \Xi_{1}$ and $A \in \Xi_{2}, \quad \Theta(C) \neq \varnothing$, and $A \in \Theta(C) \Leftrightarrow A \subseteq C$. Furthermore, the following statements are equivalent: 
(1) $\Theta$ is one-to-one from $\Xi_{1}$ onto $\Xi_{2}$;

(2) $\Theta$ is single valued and surjective;

(3) $\left|\Xi_{1}\right|=\left|\Xi_{2}\right|$

When either one of the equivalent statements in the previous proposition holds we will say that $\Theta$ is a final equivalence.

Proposition 5.12 (Combinatorics monotonicity). Let $\varphi, \psi \in \mathcal{O}(X)$ :

(1) $\left|\Lambda_{\text {final }}^{\Omega}(\varphi)\right| \geq\left|\Lambda_{\text {final }}^{R}(\varphi)\right|$ and $\left|\Sigma_{\text {final }}^{\Omega}(\varphi)\right| \geq\left|\Sigma_{\text {final }}^{R}(\varphi)\right|$;

(2) if $\varphi \leq \psi$, then $\left|\Lambda_{\text {final }}^{\Omega}(\varphi)\right| \geq\left|\Lambda_{\text {final }}^{\Omega}(\psi)\right|$ and $\left|\Sigma_{\text {final }}^{\Omega}(\varphi)\right| \geq\left|\Sigma_{\text {final }}^{\Omega}(\psi)\right|$;

(3) if $\varphi \prec \psi$, then $\left|\Lambda_{\text {final }}^{R}(\varphi)\right| \geq\left|\Lambda_{\text {final }}^{R}(\psi)\right|$ and $\left|\Sigma_{\text {final }}^{R}(\varphi)\right| \geq\left|\Sigma_{\text {final }}^{R}(\psi)\right|$;

(4) let $\Theta$ be any of the mappings, $\theta_{\varphi, \psi}, \theta_{\varphi, \psi}^{*}$ or $\tilde{\theta}_{\varphi}$; given final components $A$ and $C$, if $A \in \Theta(C)$ then the period of $C$ divides the period of $A$.

The combinatorics monotonicity above implies the following characterization of combinatorial equivalence.

Proposition 5.13. Given $\varphi, \psi \in \mathcal{O}(X)$ such that $\varphi \leq \psi$, the following statements are equivalent:

(1) $\varphi$ is combinatorially equivalent to $\psi$;

(2) $\left|\Sigma_{\text {final }}^{\Omega}(\varphi)\right|=\left|\Sigma_{\text {final }}^{\Omega}(\psi)\right|$;

(3) $\left|\Lambda_{\text {final }}^{\Omega}\left(\varphi^{d}\right)\right|=\left|\Lambda_{\text {final }}^{\Omega}\left(\psi^{d}\right)\right|$, for all $d \in \mathbb{N}$.

Proof. Assume that $\varphi \leq \psi$ and take $d$ to be a multiple of all periods of $\Omega$-final classes of $\varphi$. Then all $\Omega$-final classes of $\varphi^{d}$ are connected and $\Sigma_{\text {final }}^{\Omega}(\varphi)=\Lambda_{\text {final }}^{\Omega}\left(\varphi^{d}\right)$. Since $d$ is also a multiple of all periods of classes in $\Lambda_{\text {final }}^{\Omega}(\psi)$ we have $\Sigma_{\text {final }}^{\Omega}(\psi)=\Lambda_{\text {final }}^{\Omega}\left(\psi^{d}\right)$. These equalities show that (3) implies (2).

The next lemma and proposition follow easily from the previous propositions. 
Lemma 5.14 (Factorization lemma). In each of the factorizations of Proposition 5.10, the composition is a final equivalence if and only if both factors are final equivalences.

Proposition 5.15. Given $\varphi, \psi \in \mathcal{O}(X)$, such that $\varphi \leq \psi$, if $\theta_{\varphi, \psi}$ is a final equivalence then every map $\zeta \in \mathcal{O}(X)$ such that $\varphi \leq \zeta \leq \psi$ is combinatorially equivalent to both $\varphi$ and $\psi$.

Propositions 5.13 and 5.15 are key to the proof of the two propositions below, as well as the proof of the stability characterization Theorem 5.3.

Proposition 5.16 (Upper stability of final combinatorics). Given $\varphi \in \mathcal{O}(X)$, there is some $\epsilon>0$ such that $\theta_{\varphi, \varphi_{\epsilon}^{*}}^{*}$ is a final equivalence.

Proposition 5.17 (Lower stability of final combinatorics). Given $\varphi \in \mathcal{O}(X)$, there is some $\epsilon>0$ such that $\theta_{\varphi_{\epsilon}^{\circ}, \varphi}$ is a final equivalence.

If any of these statements holds for some $\epsilon_{0}>0$ then it also holds for all $0<\epsilon<\epsilon_{0}$. This follows from the factorization Lemma 5.14. The proofs of these two propositions will be given by the sequence of lemmas which follow below.

Lemma 5.18. Every $\varphi \in \mathcal{O}(X)$ is combinatorially equivalent to $\widehat{\varphi}$.

Proof. Given $\varphi \in \mathcal{O}(X)$, the open set $S(\varphi)=\cup_{n=1}^{\infty} \operatorname{graph}\left(\varphi^{n}\right)$ is the graph of the pre-order $\rightsquigarrow \varphi$. The intersection $E(\varphi)=S(\varphi) \cap S(\varphi)^{-1}$ is an open set which together with the diagonal $\Delta_{X} \subseteq X \times X$ gives the graph of the equivalence relation $\rightsquigarrow_{\varphi}$. Finally, denoting by $\pi: X \times X \rightarrow X$ the projection onto the first factor, we have $\Omega(\varphi)=\pi(E(\varphi))$.

By Proposition 4.3 it follows that $\operatorname{graph} \varphi^{n}$ is dense in $\operatorname{graph}(\widehat{\varphi})^{n}$, for each $n \geq 1$. Therefore $S(\varphi)$ is dense in $S(\widehat{\varphi})$, which implies that $E(\varphi)$ is dense in $E(\widehat{\varphi})$, and in turn this implies that $\Omega(\varphi)$ is dense in $\Omega(\widehat{\varphi})$. Given open sets $U_{1}, U_{2} \subseteq \Omega(\varphi)$, since $S(\varphi)$ is dense in $S(\widehat{\varphi}), U_{1} \rightsquigarrow \widehat{\varphi} U_{2}$ implies $U_{1} \rightsquigarrow{ }_{\varphi} U_{2}$. This shows that the map $\tau_{\varphi, \widehat{\varphi}}$ : $\Lambda_{\text {final }}^{\Omega}(\widehat{\varphi}) \rightarrow \mathcal{P}\left(\Lambda_{\text {final }}^{\Omega}(\varphi)\right)$ is bijective. 
Because of Proposition 4.3, we have $\varphi^{d} \leq(\widehat{\varphi})^{d} \leq \widehat{\left(\varphi^{d}\right)}$ and applying the previous argument to $\varphi^{d}$ we see that

$$
\left|\Lambda_{\text {final }}^{\Omega}\left(\varphi^{d}\right)\right|=\left|\Lambda_{\text {final }}^{\Omega}\left((\widehat{\varphi})^{d}\right)\right|=\left|\Lambda_{\text {final }}^{\Omega}\left(\widehat{\varphi^{d}}\right)\right|,
$$

for every $d \in \mathbb{N}$. By Proposition 5.13 this implies that $\varphi$ and $\widehat{\varphi}$ are combinatorially equivalent.

Corollary 5.19. All open maps in a class $[\varphi]$ of the quotient semigroup $\mathbb{O}(X)$ are combinatorially equivalent to each other.

Let $\varphi \in \mathcal{O}(X)$. We will call the thickness of $\varphi$ the smallest volume ( $m$-measure) of all components in $\Sigma_{\text {final }}^{\Omega}(\varphi)$. We say that an open set $K \subseteq \Omega_{\text {final }}(\varphi)$ is a final kernel of $\varphi$ if and only if there is a one-to-one correspondence $C \mapsto K_{C}$, between components $C \in \Sigma_{\text {final }}^{\Omega}(\varphi)$ and connected components $K_{C}$ of $K$, such that $\overline{K_{C}} \subseteq C$ for every $C \in \Sigma_{\text {final }}^{\Omega}(\varphi)$. We say that $K$ is a final kernel with finite order $N$ if and only if $K$ is a final kernel of $\varphi$, and furthermore

(1) For each component $C \in \Sigma_{\text {final }}^{\Omega}(\varphi)$ of period $d$, the only connected component $K_{C}$ of $K$ contained in $C$ satisfies $\overline{C \times K_{C}} \subseteq \operatorname{graph} \varphi^{N d}$.

(2) For each $x \in X, \varphi^{N}(x)$ contains at least the closure of one of $K$ 's connected components.

We will call the thickness of the final kernel $K$ the smallest volume of all connected components of $K$.

Lemma 5.20. Given $\varphi \in \mathcal{O}(X)$, every final kernel $K$ of $\varphi$ is a final kernel with some finite order $N \in \mathbb{N}$. In particular, $\varphi$ admits finite order final kernels, whose thickness is arbitrarily close to the thickness of $\varphi$.

Proof. Let $K$ be a final kernel of $\varphi$. Let $C \in \Sigma_{\text {final }}^{\Omega}(\varphi)$ be a connected component of period $d$ and let $K_{C}$ be the correspondent connected component of $K$. Fix $x_{0} \in C$. There is $n \in \mathbb{N}$ such that $x_{0} \in \varphi^{n}\left(x_{0}\right)$ and all such $n$ 's are multiples of $d$. It is clear that if $x_{0} \in \varphi^{n}\left(x_{0}\right)$ and $x_{0} \in \varphi^{m}\left(x_{0}\right)$, then $x_{0} \in \varphi^{n+m}\left(x_{0}\right)$. Consequently a simple number theory argument ensures that, for some large enough $n_{0}$ and for all 
$n \geq n_{0}$, one has $x_{0} \in \varphi^{n d}\left(x_{0}\right)$. For any $(x, y) \in \bar{C} \times C$ there is $n \in \mathbb{N}$ such that $y \in \varphi^{n}(x)$ and all such $n$ are multiples of $d$. Furthermore, because $\varphi$ is open, the map $n(x, y)=\inf \left\{n: y \in \varphi^{n}(x)\right\}$ is upper semi-continuous in $(x, y) \in \bar{C} \times C$. Therefore, there exists $N_{0}=N_{0}\left(K_{C}\right)$ such that if $(x, y) \in \bar{C} \times \overline{K_{C}}$ then $x_{0} \in \varphi^{k}(x)$ and $y \in \varphi^{l}\left(x_{0}\right)$, for some $k, l<N_{0}$. Set $N_{C}$ to be the integral part of $n_{0}+2 N_{0} / d+1$. Then for every $n \geq N_{C}$, one has $y \in \varphi^{n d}(x)$, that is, $\bar{C} \times \overline{K_{C}} \subseteq \operatorname{graph} \varphi^{n d}$ for every $n \geq N_{C}$. Set $N_{1}=\max \left\{N_{C}: C \in \Sigma_{\text {final }}^{\Omega}(\varphi)\right\}$. Then for every $N \geq N_{1}$ and for each component $C \in \Sigma_{\text {final }}^{\Omega}(\varphi)$ of period $d$, one has $\bar{C} \times \overline{K_{C}} \subseteq \operatorname{graph} \varphi^{N d}$, which proves item (1) in the definition of final kernel of order $N$.

For any $x \in X$ define $n(x)=\inf \left\{n: \varphi^{n}(x) \cap \Omega_{\text {final }}(\varphi) \neq \varnothing\right\}$. The map $n: X \rightarrow \mathbb{N}$ is bounded because $X=W_{\Omega}^{\mathrm{s}}\left(\Omega_{\mathrm{final}}(\varphi)\right)$, and is upper semi-continuous because $\varphi$ is open. Furthermore, as $\Omega_{\text {final }}(\varphi)$ is $\varphi$-invariant, if for some $n_{0}$ one has $\varphi^{n_{0}}(x) \cap \Omega_{\text {final }}(\varphi) \neq \varnothing$, then $\varphi^{n}(x) \cap \Omega_{\text {final }}(\varphi) \neq \varnothing$ for every $n \geq n_{0}$. Altogether these facts ensure that there exists $N_{2} \in \mathbb{N}$ such that, for each $x \in X, \varphi^{N_{2}}(x) \cap \Omega_{\text {final }}(\varphi) \neq \varnothing$. Therefore, for each $x \in X$ there exists $C \in \Sigma_{\text {final }}^{\Omega}(\varphi)$ such that $\varphi^{N_{2}}(x) \cap C \neq \varnothing$. From above we know that $\bar{C} \times \overline{K_{C}} \subseteq \operatorname{graph} \varphi^{N_{1} d}$. Therefore, setting $N=N_{1} d+N_{2}$ one has that, for each $x \in X$, $\varphi^{N}(x)$ contains at least the closure of one of $K$ 's connected components. This proves item (2) in the definition of final kernel of finite order $N$. Thus $K$ is a final kernel of order $N$.

Proof. (of Proposition 5.17) Take some final kernel $K \subseteq \Omega_{\text {final }}(\varphi)$ and choose $N \in \mathbb{N}$ such that $K$ is a finite kernel of order $N$. It is clear that for small enough $\epsilon>0$, $K \subseteq \Omega_{\text {final }}\left(\varphi_{\epsilon}^{\circ}\right)$ is also a final kernel of the same order $N$ for the map $\varphi_{\epsilon}^{\circ}$. We claim that for such $\epsilon, \theta_{\varphi_{\epsilon}^{\circ}, \varphi}$ is a final equivalence. Indeed, item (1) in the definition of final kernel implies that for a given component $C \in \Sigma_{\text {final }}^{\Omega}(\varphi)$ there is at most one component $C_{\epsilon} \in \Sigma_{\text {final }}^{\Omega}\left(\varphi_{\epsilon}^{\circ}\right)$ contained in $C$ : the component that contains $K_{C}$. On the other hand, item (2) of the same definition implies that any final component $C_{\epsilon} \in \Sigma_{\text {final }}^{\Omega}\left(\varphi_{\epsilon}^{\circ}\right)$ must contain one of $K$ 's components and, therefore, be contained inside a final component $C \in \Sigma_{\text {final }}^{\Omega}(\varphi)$. These two facts together show that $\theta_{\varphi_{\epsilon}^{\circ}, \varphi}$ is a final equivalence.

Lemma 5.21. Given $\varphi \in \mathcal{O}(X)$, there is some $\epsilon>0$ such that $\tau_{\varphi, \varphi_{\epsilon}^{*}}^{*}$ is a final equivalence.

Proof. Since for any pair $\left(A_{1}, A_{2}\right)$ of classes $A_{1}, A_{2} \in \Lambda_{\text {final }}^{R}(\varphi)$, we have $A_{1} \vdash_{\varphi} A_{2}$, we can choose $\epsilon>0$ small enough so that for all those pairs $A_{1} \varkappa_{\varphi_{\epsilon}^{*}} A_{2}$. 
We have to prove that any final class $A \in \Lambda_{\text {final }}^{R}(\varphi)$ is contained in some final class $C \in \Lambda_{\text {final }}^{\Omega}\left(\varphi_{\epsilon}^{*}\right)$. Given $A \in \Lambda_{\text {final }}^{R}(\varphi)$ clearly there is some $C \in \Lambda_{\text {final }}^{\Omega}\left(\varphi_{\epsilon}^{*}\right)$ such that $A \rightsquigarrow \varphi_{\epsilon}^{*} C$, and for this class $C$ there is $A^{\prime} \in \Lambda_{\text {final }}^{R}(\varphi)$ such that $A^{\prime} \subseteq C$. Therefore, $A \rightsquigarrow \varphi_{\epsilon}^{*} A^{\prime}$, which by choise of $\epsilon$ implies $A \rightarrow_{\varphi} A^{\prime}$, and so, as $A$ is a final class, $A=A^{\prime}$.

Finally we have to see that given $C \in \Lambda_{\text {final }}^{\Omega}\left(\varphi_{\epsilon}^{*}\right)$ there is at most one class $A \in$ $\Lambda_{\text {final }}^{R}(\varphi)$ contained in $C$. Assume $A_{1}, A_{2} \in \Lambda_{\text {final }}^{R}(\varphi)$ are two final classes contained inside $C \in \Lambda_{\text {final }}^{\Omega}\left(\varphi_{\epsilon}^{*}\right)$. Then $A_{1} \rightsquigarrow_{\varphi_{\epsilon}^{*}} A_{2}$ and $A_{2} \rightsquigarrow_{\varphi_{\epsilon}^{*}} A_{1}$, which by choice of $\epsilon$ implies $A_{1} \rightarrow_{\varphi} A_{2}$ and $A_{2} \rightarrow_{\varphi} A_{1}$. This proves that $A_{1}=A_{2}$.

Lemma 5.22. There is $\epsilon_{0}>0$ such that $\left\{\Omega_{\text {final }}\left(\varphi_{\epsilon}^{*}\right)\right\}_{0<\epsilon<\epsilon_{0}}$ is an outer approximation of $R_{\text {final }}(\varphi)$.

Proof. Take $\epsilon_{0}>0$ given by Lemma 5.21. Then for $0<\epsilon<\epsilon_{0}, \quad R_{\text {final }}(\varphi) \subseteq \Omega_{\text {final }}\left(\varphi_{\epsilon}^{*}\right)$. Since it is clear that $\left\{\Omega\left(\varphi_{\epsilon}^{*}\right)\right\}_{0<\epsilon<\epsilon_{0}}$ is an outer approximation of $R(\varphi)$, the lemma follows.

Proof. (of Proposition 5.16) Let $\gamma>0$ be the minimum of all distances between components in $\Sigma_{\text {final }}^{R}(\varphi)$. Take $\epsilon>0$ small enough so that $\tau_{\varphi, \varphi_{\epsilon}^{*}}^{*}$ is a final equivalence and $R_{\text {final }}(\varphi) \subseteq \Omega_{\text {final }}\left(\varphi_{\epsilon}^{*}\right) \subseteq B_{\gamma / 2}\left(R_{\text {final }}(\varphi)\right)$. It follows that each connected component of $R_{\text {final }}(\varphi)$ is contained inside a connected component of $\Omega_{\text {final }}\left(\varphi_{\epsilon}^{*}\right)$, and each connected component of $\Omega_{\text {final }}\left(\varphi_{\epsilon}^{*}\right)$ contains at most one connected component of $R_{\text {final }}(\varphi)$. Therefore $\theta_{\varphi, \varphi_{\varepsilon}^{*}}^{*}$ is a final equivalence.

We say that $\varphi \in \mathcal{O}(X)$ satisfies the combinatorial stability condition if and only if $\tilde{\theta}_{\varphi}$ is a final equivalence, or equivalently if and only if $\left|\Lambda_{\text {final }}^{\Omega}(\varphi)\right|=\left|\Lambda_{\text {final }}^{R}(\varphi)\right|$ and $\left|\Sigma_{\text {final }}^{\Omega}(\varphi)\right|=\left|\Sigma_{\text {final }}^{R}(\varphi)\right|$.

Theorem 5.3 (Stability characterization). Let $\mathcal{O}_{1}$ be any topological semigroup of open maps. For any $\varphi \in \mathcal{O}_{1}, \varphi$ is combinatorially stable in $\mathcal{O}_{1}$ if and only if $\varphi$ satisfies the combinatorial stability condition.

Proof. In view of Corollary 5.19 it is enough proving this theorem for the quotient semigroup $\mathbb{O}_{1}=\mathcal{O}_{1} / \sim$.

Given $\varphi \in \mathbb{O}_{1}$ assume that $\tilde{\theta}_{\varphi}$ is a final equivalence. Let $K \subseteq \Omega_{\text {final }}(\varphi)$ be some final kernel with finite order $N \in \mathbb{N}$ and positive thickness $c>0$. Let $K_{1}, \cdots, K_{p}$ 
be the connected components of $K$, and $C_{1}, \cdots, C_{p}$ be the correspondent components in $\Sigma_{\text {final }}^{\Omega}(\varphi)$. Take $0<\epsilon<c / 3$ as in Proposition 5.16. Find an open cover of $X$, $\left\{U_{i}: 1 \leq i \leq p\right\}$, such that $\overline{U_{i} \times K_{i}} \subseteq \operatorname{graph}\left(\varphi^{N}\right)$ for every $i=1, \cdots, p$. Notice that, since $K$ is a final kernel of order $N$, we have $\overline{C_{i} \times K_{i}} \subseteq \operatorname{graph}\left(\varphi^{N d_{i}}\right)$, where $d_{i}$ is the period of $C_{i}$. Then choose some neighbourhood $\mathcal{N}$ of $\varphi$ in $\mathbb{O}_{1}$ such that for every $\psi \in \mathcal{N}, \rho_{H}(\psi, \varphi)<\epsilon, m\left(K_{i} \backslash \psi^{N}(x)\right)<\epsilon$ if $x \in \bar{U}_{i}$, and $m\left(K_{i} \backslash \psi^{N d_{i}}(x)\right)<\epsilon$ when $x \in \bar{C}_{i}$, for $i=1, \cdots, p$. Such a neighbourhood $\mathcal{N}$ exists by Definition 4.2. Take any $\psi$ in $\mathcal{N}$. Inequality $\rho_{H}(\psi, \varphi)<\epsilon$ implies $\psi \leq \varphi_{\epsilon}^{*}$, and so we may consider the mapping $\theta_{\epsilon}=\theta_{\psi, \varphi_{\epsilon}^{*}}: \Sigma_{\text {final }}^{\Omega}\left(\varphi_{\epsilon}^{*}\right) \rightarrow \mathcal{P}\left(\Sigma_{\text {final }}^{\Omega}(\psi)\right)$. Let us show now that $\theta_{\epsilon}$ is a final equivalence, which, in view of $\epsilon$ choice, proves that $\psi$ is combinatorially equivalent to $\varphi$. Given $B \in \Sigma_{\text {final }}^{\Omega}(\psi)$, pick an index $i=1, \cdots, p$ such that $B \cap U_{i} \neq \varnothing$ and take $x \in B \cap U_{i}$. Because $m\left(K_{i} \backslash \psi^{N}(x)\right)<\epsilon<m\left(K_{i}\right) / 3$, we have $m\left(K_{i} \cap \psi^{N}(x)\right)>2 m\left(K_{i}\right) / 3$, which implies $\psi^{N}(x) \cap K_{i} \neq \varnothing$. So there is some $z \in K_{i} \subseteq \Omega_{\text {final }}\left(\varphi_{\epsilon}^{*}\right)$ such that $B \stackrel{\sim}{*} z$. Thus, since $\psi \leq \varphi_{\epsilon}^{*}, \quad B \subseteq \Omega_{\mathrm{final}}\left(\varphi_{\epsilon}^{*}\right)$. This proves, denoting by $C$ the $\Omega$-final component of $\varphi_{\epsilon}^{*}$ containing $z$, that $B \in \theta_{\epsilon}\left(\left(\pi_{\varphi_{\epsilon}^{*}}\right)^{-N}(C)\right.$. Therefore $\theta_{\epsilon}$ is surjective. Let now $B_{1}, B_{2} \in \Sigma_{\text {final }}^{\Omega}(\psi)$ be two components such that $B_{1}, B_{2} \in \theta_{\epsilon}\left(\tilde{C}_{j}\right)$ where for some $j=1, \cdots, p, \tilde{C}_{j} \in \sum_{\text {final }}^{\Omega}\left(\varphi_{\epsilon}^{*}\right)$ is the unique component that contains $C_{j}$. Since $K_{j} \subseteq C_{j} \subseteq \tilde{C}_{j}$, arguing as before, for each $i=1,2$, there is some point $x_{i} \in K_{j}$ such that $B_{i} \rightsquigarrow \psi x_{i}$. Since these are final components, we may choose the points $x_{i}$ so that $x_{i} \in B_{i}$. Because $x_{1}, x_{2} \in K_{j} \subseteq C_{j}$, by the choice of neighbourhood $\mathcal{N}$, we have

$$
m\left(K_{j} \backslash \psi^{N d_{j}}\left(x_{1}\right)\right)<m\left(K_{j}\right) / 3 \quad \text { and } \quad m\left(K_{j} \backslash \psi^{N d_{j}}\left(x_{2}\right)\right)<m\left(K_{j}\right) / 3,
$$

and so

$$
m\left(K_{j} \cap \psi^{N d_{j}}\left(x_{1}\right) \cap \psi^{N d_{j}}\left(x_{2}\right)\right) \geq m\left(K_{j}\right) / 3,
$$

wich implies that $\psi^{N d}\left(x_{1}\right) \cap \psi^{N d}\left(x_{2}\right) \neq \varnothing$. Since $B_{1}$ and $B_{2}$ are final components, it follows that $B_{1}=B_{2}$. This proves that $\theta_{\epsilon}$ is single valued and, therefore, a final equivalence.

Assume now that $\varphi$ is $\mathbb{O}_{1}$-combinatorially stable and let $\mathcal{N}$ be a neighbourhood of $\varphi$ in $\mathbb{O}_{1}$ where all open maps are combinatorially equivalent. Take $\epsilon>0$ as in Proposition 5.16. By item (b) of Definition 3.3, used in Definition 4.2, there is an outer approximation $\left\{\varphi_{n}\right\}$ of $\varphi$ such that $\lim _{n \rightarrow \infty} \varphi_{n}=\varphi$. Then for all large enough $n, \varphi_{n} \in \mathcal{N}$ and $\varphi_{n} \prec \varphi_{\epsilon}^{*}$. Therefore $\theta_{\varphi, \varphi_{n}}$ is a final equivalence, because $\varphi$ and $\varphi_{n}$ are combinatorially equivalent; and $\theta_{\varphi, \varphi_{n}}^{*}$ is also a final equivalence, by the choice of $\epsilon$ in Proposition 5.16. Since $\theta_{\varphi, \varphi_{n}}=\tilde{\theta}_{\varphi} \circ \theta_{\varphi, \varphi_{n}}^{*}$, the Factorization Lemma 5.10 implies that $\tilde{\theta}_{\varphi}$ is a final equivalence. 
Theorem 5.4 (Genericity of combinatorial stability). Let $\mathcal{O}_{1}$ be any topological semigroup of open maps. The set of $\mathcal{O}_{1}$-combinatorially stable maps is open and dense in the semigroup $\mathcal{O}_{1}$.

Proof. By definition, combinatorial stability is an open property. Thus it is enough to prove density. Consider any open map $\varphi \in \mathcal{O}_{1}$. Take $\epsilon>0$ as in Proposition 5.16. By Definition 3.3(b), used in Definition 4.2, there is an outer approximation $\left\{\varphi_{n}\right\}$ of $\varphi$ such that $\lim _{n \rightarrow \infty} \varphi_{n}=\varphi$. Then for all large enough $n, \varphi_{n} \prec \varphi_{\epsilon}^{*}$, and since $\theta_{\varphi, \varphi_{\varepsilon}^{*}}^{*}=\theta_{\varphi, \varphi_{n}}^{*} \circ \tilde{\theta}_{\varphi_{n}} \circ \theta_{\varphi_{n}, \varphi_{\varepsilon}^{*}}^{*}$, the Factorization lemma 5.10 implies that $\tilde{\theta}_{\varphi_{n}}$ is a final equivalence. Thus, $\varphi$ is the limit of the combinatorially stable open maps $\varphi_{n}$.

We say that an $\Omega$-final class of $\varphi$ is unstable when it is contained inside an $R$-class of $\varphi$ which is not final.

We say that two or more $\Omega$-final classes $C_{1}, \cdots, C_{k}$ of $\varphi$ colapse together when they are contained in the same $R$-final class of $\varphi$.

We say that the period of an $\Omega$-final class $C$ of $\varphi$ is colapsing when it is contained in one $R$-final class whose period is less than the period of $C$.

The following proposition describes the bifurcation set of $\varphi$ in a topological semigroup of open maps. It's just an obvious reformulation of Theorem 5.3.

Proposition 5.23. Let $\mathcal{O}_{1}$ be any topological semigroup of open maps. Given $\varphi \in \mathcal{O}_{1}$, $\varphi$ is not $\mathcal{O}_{1}$-combinatorially stable if and only if one of the following alternatives hold:

(a) one $\Omega$-final class of $\varphi$ is unstable;

(b) two or more $\Omega$-final classes of $\varphi$ colapse together;

(c) the period of one $\Omega$-final class $C$ of $\varphi$ is colapsing.

Recall that a space is called a Baire space if and only if any countable intersection of open and dense subsets is still dense. By Baire theorem, any complete metric space is a Baire space. In particular, compact metric spaces are Baire spaces. It is also straightforward to check that an inductive limit of Baire spaces is again a Baire space. Therefore since the topological semigroup $\mathbb{O}_{\text {Lip }}^{r}$ is the inductive limit of compact metric 
spaces, it is a Baire space. On Baire spaces, a countable intersection of open and dense subsets is called a residual subset.

Let us say that an open map $\varphi \in \mathcal{O}(X)$ satisfies the topological stability condition if and only if $\varphi$ satisfies the combinatorial stability condition, $\overline{\Omega_{\text {final }}(\varphi)}=R_{\text {final }}(\varphi)$ and both these sets are Jordan measurable. Then set

$$
\mathcal{O}_{\text {stable }}=\{\varphi \in \mathcal{O}(X): \varphi \quad \text { satisfies the topological stability condition }\} .
$$

We say that a closed set $K \subseteq X$ has regular boundary if $\partial K=\partial\left(K^{\circ}\right) \Leftrightarrow K=\overline{K^{\circ}}$.

Lemma 5.24. For every $\varphi \in \mathcal{O}_{\text {Cont }}(X)$, the closed set $R_{\text {final }}(f)$ has regular boundary.

Proof. It is obvious that the closure of an open set is always a closed set with regular boundary. Then for any open map $\varphi \in \mathcal{O}_{\text {Cont }}(X)$, and any $x \in X, \bar{\varphi}(x)=\overline{\varphi(x)}$ has regular boundary. The equality holds because $\varphi$ is continuous. Thus, for each class $C \in \Lambda_{\text {final }}^{R}(\varphi), \quad C=\bar{\varphi}(C)=\overline{\varphi(C)}$, which proves that $C$, being the closure of the open set $\varphi(C)$, has regular boundary. Since $\Lambda_{\text {final }}^{R}(\varphi)$ is finite, $R_{\text {final }}(\varphi)$ is a finite disjoint union of closed sets with regular boundary, and therefore has itself regular boundary.

Lemma 5.25. Given $\varphi \in \mathcal{O}_{\text {Cont }}(X)$, let $\left\{\varphi_{\epsilon}^{*}\right\}_{0<\epsilon<\epsilon_{0}}$ be any outer approximation of $\varphi$ by continuous open maps. Then the set $\{\epsilon \in] 0, \epsilon_{0}\left[: \varphi_{\epsilon}^{*} \notin \mathcal{O}_{\text {stable }}\right\}$ is at most countable. In particular, in any topological semigroup of open maps $\mathcal{O}_{1} \subseteq \mathcal{O}_{\text {Cont }}(X)$, the set $\mathcal{O}_{1} \cap \mathcal{O}_{\text {stable }}$ is dense in $\mathcal{O}_{1}$.

A similar result holds for inner approximations.

Proof. Let $A_{\epsilon}=R_{\text {final }}\left(\varphi_{\epsilon}^{*}\right)^{\circ} \backslash \overline{\Omega_{\text {final }}\left(\varphi_{\epsilon}^{*}\right)}$ and $B_{\epsilon}=R_{\text {final }}\left(\varphi_{\epsilon}^{*}\right) \backslash \Omega_{\text {final }}\left(\varphi_{\epsilon}^{*}\right)$ for each $0<\epsilon<\epsilon_{0}$. Then $\left\{A_{\epsilon}\right\}_{\epsilon>0}$ is a family of pairwise disjoint open sets, and $\left\{B_{\epsilon}\right\}_{\epsilon>0}$ is a family of pairwise disjoint closed sets. By monotonicity of the combinatorics, see Proposition 5.12, the set $\Xi_{0}=\{\epsilon \in] 0, \epsilon_{0}\left[: \tilde{\theta}_{\varphi_{\epsilon}^{*}}\right.$ is not a final equivalence $\}$ is at most finite. Since the topology of $X$ admits countable open sub-basis, the set $\Xi_{1}=\{\epsilon \in$ ] $0, \epsilon_{0}\left[: A_{\epsilon} \neq \varnothing\right\}$ is at most countable. Because $\sum_{0<\epsilon<\epsilon_{0}} m\left(B_{\epsilon}\right) \leq m(X)=1$, the set $\Xi_{2}=\{\epsilon \in] 0, \epsilon_{0}\left[: m\left(B_{\epsilon}\right)>0\right\}$ is, once more, at most countable. Finally, it should be clear that the set $\{\epsilon \in] 0, \epsilon_{0}\left[: \varphi_{\epsilon}^{*} \notin \mathcal{O}_{\text {stable }}\right\}$ is covered by the countable union $\Xi_{0} \cup \Xi_{1} \cup \Xi_{2}$. At this point we use the previous lemma to ensure that $A_{\epsilon}=\varnothing$ implies $R_{\text {final }}\left(\varphi_{\epsilon}^{*}\right)=\overline{\Omega_{\text {final }}\left(\varphi_{\epsilon}^{*}\right)}$. 
Theorem 5.5 (Continuity of the attractors). Let $\mathbb{O}_{1}$ be any topological semigroup of open maps.

(1) The mappings $\varphi \mapsto \Omega_{\text {final }}(\varphi)$ from $\mathbb{O}_{1}$ into $\left(\mathbb{U}(X), \rho_{H}\right)$, and $\varphi \mapsto m\left(\Omega_{\text {final }}(\varphi)\right)$ from $\mathbb{O}_{1}$ into $\mathbb{R}$ are lower semi-continuous.

(2) The mappings $\varphi \mapsto R_{\text {final }}(\varphi)$ from $\mathbb{O}_{1}$ into $\left(\mathbb{K}(X), \rho_{H}\right)$, and $\varphi \mapsto m\left(R_{\text {final }}(\varphi)\right)$ from $\mathbb{O}_{1}$ into $\mathbb{R}$ are upper semi-continuous.

(3) The four mappings on $\mathbb{O}_{1}, \varphi \mapsto \Omega_{\text {final }}(\varphi), \varphi \mapsto R_{\text {final }}(\varphi), \varphi \mapsto m\left(R_{\text {final }}(\varphi)\right)$ and $\varphi \mapsto m\left(\Omega_{\text {final }}(\varphi)\right)$ are continuous at all $\varphi \in \mathcal{O}_{1} \cap \mathcal{O}_{\text {stable }}(X)$.

(4) The final components in $\Sigma_{\text {final }}^{\Omega}(\varphi)$ depend continuously on $\varphi$ at those open maps

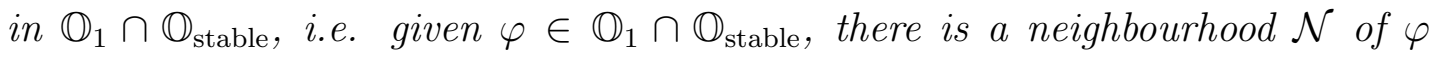
in $\mathbb{O}_{1}$, and for each $\psi \in \mathcal{N}$ there is a conjugation $h_{\varphi, \psi}: \Sigma_{\text {final }}^{\Omega}(\varphi) \rightarrow \Sigma_{\text {final }}^{\Omega}(\psi)$ between $\pi_{\varphi}$ and $\pi_{\psi}$ such that $\lim \rho_{H}\left(h_{\varphi, \psi}(C), C\right)=0$, as $\psi$ tends to $\varphi$, for every component $C \in \Sigma_{\text {final }}^{\Omega}(\varphi)$.

Proof. Given $\varphi \in \mathbb{O}_{1}$ let $K$ be a final kernel of $\varphi$ such that $B_{\epsilon}(K) \supseteq \Omega_{\text {final }}(\varphi)$ and $m(K) \geq m\left(\Omega_{\text {final }}(\varphi)\right)-\epsilon$. Let $K_{1}, K_{2}, \cdots, K_{p}$ be the connected components of $K$, and $C_{1}, C_{2}, \cdots, C_{p}$ be the corresponding components in $\Sigma_{\text {final }}^{\Omega}(\varphi)$. Denote by $d_{i}$ the period of component $C_{i}$, for $i=1, \cdots, p$. Choose $N \in \mathbb{N}$ such that kernel $K$ has order $N$. Arguing as in the proof of Theorem 5.3, we can find a neighbourhood $\mathcal{N}$ of $\varphi$ such that for each $\psi \in \mathcal{N}$ and $i=1, \cdots, p, K_{i} \cap \Omega_{\text {final }}(\psi) \neq \varnothing$. By Proposition 4.8 we can make $\mathcal{N}$ smaller so that for every $\psi \in \mathcal{N}$, every $i=1, \cdots, p$, and each $x \in K_{i}$, one has $B_{\epsilon}\left(\psi^{N d_{i}}(x)\right) \supseteq \varphi^{N d_{i}}(x)$. By Definition 4.2 we make $\mathcal{N}$ even smaller so that for every $\psi \in \mathcal{N}$, every $i=1, \cdots, p$, and each $x \in C_{i}$, one has $m\left(K_{i} \backslash \psi^{N d_{i}}(x)\right)<\epsilon / p$. Now pick any $\psi \in \mathcal{N}$. For each $i=1, \cdots, p$ choose $x_{i} \in K_{i} \cap \Omega_{\text {final }}(\psi)$. Then

$$
B_{\epsilon}\left(\Omega_{\text {final }}(\psi)\right) \supseteq \cup_{i=1}^{p} B_{\epsilon}\left(\psi^{N d_{i}}\left(x_{i}\right)\right) \supseteq \cup_{i=1}^{p} \varphi^{N d_{i}}\left(x_{i}\right) \supseteq \cup_{i=1}^{p} K_{i}=K
$$

And so

$$
B_{2 \epsilon}\left(\Omega_{\text {final }}(\psi)\right) \supseteq B_{\epsilon}(K) \supseteq \Omega_{\text {final }}(\varphi)
$$


Analogously

$$
\begin{aligned}
m\left(\Omega_{\text {final }}(\psi)\right) & \geq \sum_{i=1}^{p} m\left(\psi^{N d_{i}}\left(x_{i}\right) \cap K_{i}\right) \\
& =\sum_{i=1}^{p} m\left(K_{i}\right)-m\left(K_{i} \backslash \psi^{N d_{i}}\left(x_{i}\right)\right) \\
& >m(K)-\sum_{i=1}^{p} \epsilon / p=m(K)-\epsilon \\
& \geq m\left(\Omega_{\text {final }}(\varphi)\right)-2 \epsilon .
\end{aligned}
$$

This proves the lower continuity of both mappings and establishes Theorem 5.5(1). The upper continuity in Theorem 5.5(2) follows from Lemma 5.22. Theorem 5.5(3) follows easily from (1) and (2). Finally, (4) follows from (3).

Theorem 5.6. Given any topological semigroup of open maps $\mathbb{O}_{1} \subseteq \mathbb{O}_{\text {Cont }}$, the set $\mathbb{O}_{1} \cap \mathbb{O}_{\text {stable }}$ is a countable intersection of open and dense subsets of $\mathbb{O}_{1}$. In particular, for each $r \geq 0, \mathbb{O}_{\text {stable }} \cap \mathbb{O}_{\text {Lip }}^{r}$ is residual in $\mathcal{O}_{\text {Lip }}^{r}$.

Proof. For each $\epsilon>0$ set

$$
\mathbb{O}_{1}^{\epsilon}=\left\{\varphi \in \mathbb{O}_{1}: B_{\epsilon}\left(\Omega_{\text {final }}(\varphi)\right) \supseteq R_{\text {final }}(\varphi) \text { and } m\left(\Omega_{\text {final }}(\varphi)\right) \geq m\left(R_{\text {final }}(\varphi)\right)-\epsilon\right\} .
$$

By Lemma 5.25, $\mathbb{O}_{1}^{\epsilon}$ is dense in $\mathbb{O}_{1}$. Theorem 5.5 proves that each $\mathbb{O}_{1}^{\epsilon}$ is an open set in $\mathbb{O}_{1}$. Therefore $\mathbb{O}_{1} \cap \mathbb{O}_{\text {stable }}=\cap_{n=1}^{\infty} \mathbb{O}_{1}^{\frac{1}{n}}$ is a countable intersection of open and dense subsets of $\mathbb{O}_{1}$.

\section{Combinatorial Stability of Continuous Maps}

Throughout this section we will denote by $\mathcal{C}^{0}(X)$ the space of all continuous mappings $f: X \rightarrow X$, with the usual $C^{0}$ topology of uniform convergence. This topology is defined by the metric

$$
d_{H}(f, g)=\max _{x \in X} d(f(x), g(x))=\sup _{x \in X} \rho_{H}(\{f(x)\},\{g(x)\}),
$$


corresponding to distance between point-set maps introduced in Definition 4.3. Let $f \in \mathcal{C}^{0}(X)$. Given $\epsilon>0, \quad f_{\epsilon}^{*}$ will denote the $\epsilon$-explosion of $f$, which is defined by $\operatorname{graph}\left(f_{\epsilon}^{*}\right)=B_{\epsilon}(\operatorname{graph} f)$. Any sequence $x_{0}, x_{1}, \cdots, x_{n} \in X$ such that $x_{i} \in f_{\epsilon}^{*}\left(x_{i-1}\right)$ for $i=1, \cdots, n$ will be called an $\epsilon$-pseudo-orbit of $f$, and we will say that $x_{n}$ is an $\epsilon$-pseudo-iterate of the state $x_{0}$. If $y$ is an $\epsilon$-pseudo-iterate of $x$ for every $\epsilon>0, y$ is said a pseudo-iterate of $x$, and we will write $x \rightarrow_{f} y$. Clearly $\rightarrow_{f}$ is a transitive closed relation. Given subsets $A, B \subseteq X$, we will write $A \rightarrow_{f} B$ when $x \rightarrow_{f} y$ for some $x \in A$ and some $y \in B$.

As usual, a subset $A \subseteq X$ is said to be $f$-invariant if and only if $f(A) \subseteq A$. An invariant set $A \subseteq X$ is called chain transitive if and only if $x \rightarrow_{f} y$ for every $x, y \in A$. We will say that $A \subseteq X$ is an acyclic attractor if and only if $A$ is a compact connected, $f$-invariant, chain transitive set and admits an isolating open neighbourhood $U \supseteq A$, in the sense that

$$
\overline{f(U)} \subseteq U \quad \text { and } \quad A=\bigcap_{n=1}^{\infty} f^{n}(U) .
$$

When the set $A$ splits as a disjoint union of $d$ compact connected sets,

$$
A=A_{0} \cup f\left(A_{0}\right) \cup \cdots f^{d}\left(A_{0}\right)
$$

such that $A_{0}$ is an acyclic attractor for $f^{d}$ we say that $A$ is a periodic attractor of period $d$. We define the basin of attraction of an attractor $A$, respectively the pseudo-basin of attraction, as $W^{\mathrm{s}}(A, f)=\bigcup_{n=0}^{\infty} f^{-n}(A)$, respectively $W_{R}^{\mathrm{s}}(A, f)=\left\{x \in X: x \rightarrow_{f}\right.$ $A\}$. Finally, we say that the map $f$ has finitely many attractors if and only if there is a finite number $p \in \mathbb{N}$ of periodic attractors $A_{1}, \cdots, A_{p}$ whose pseudo-basins of attraction cover the whole manifold, $X=\cup_{i=1}^{p} W_{R}^{\mathrm{s}}\left(A_{i}, f\right)$.

We define the chain recurrent set of $f$, and denote it by $R(f)$, as the set of all states $x \in X$ such that $x$ is a pseudo-iterate of $x$, that is,

$$
R(f)=\left\{x \in X: x \rightarrow_{f} x\right\} .
$$

This set is closed in $X$, and the closed relation $\rightarrow_{f}$ is a preorder on $R(f)$. Elements in $R(f)$ will be called chain recurrent states. Of course all points of an attractor are chain recurrent. The recurrent set $R(f)$ can be decomposed into classes defined by the following equivalence relation: $x \leftrightharpoons_{f} y \Leftrightarrow x \rightarrow_{f} y$ and $y \rightarrow_{f} x$, for any points $x, y \in R(f)$. Since $\rightarrow_{f}$ is a preorder on $R(f), \leftrightharpoons_{f}$ is an equivalence relation. The 
equivalence classes of recurrent states will be called $R$-classes. Clearly $R$-classes are closed subsets of $X$. As in the previous section, $\Lambda^{R}(f)$ will denote the set of all $R$ classes of $f$. The relation $\rightarrow_{f}$ is a partial ordering on $\Lambda^{R}(f)$. The maximal elements in $\Lambda^{R}(f)$ under $\rightarrow_{f}$ will be called $R$-final classes, i.e. $D \in \Lambda^{R}(f)$ is an $R$-final class if and only if for all $C \in \Lambda^{R}(f), D \rightarrow_{f} C$ implies $C=D$. As before, $\Lambda_{\text {final }}^{R}(f)$ will denote the set of all $R$-final classes of $f$. A chain recurrent state $x$ will be called a final chain recurrent state if every pseudo-iterate of $x$ still has some pseudo-iterate which comes back to $x$. We will call the final chain recurrent set the set, denoted by $R_{\text {final }}(f)$, of all final chain recurrent states, that is,

$$
R_{\text {final }}(f)=\left\{x \in R(f): \text { for every } y \in X, x \rightarrow_{f} y \Rightarrow y \rightarrow_{f} x\right\} .
$$

This set is, in some sense, the union of system attractors. The next proposition clears the relation between attractors on one hand, and $R$-final classes and $R_{\text {final }}(f)$ on the other.

Proposition 6.1. The set $R_{\text {final }}(f)$ has the following characterization.

(1) $R_{\text {final }}(f)$ is the union of all $R$-final classes $C \in \Lambda_{\text {final }}^{R}(f)$, and each such class is fully $f$-invariant, i.e. $f(C)=C$.

(2) The attractors of $f$ are the isolated $R$-final classes with finitely many connected components. More precisely, a subset $A \subseteq X$ is a periodic attractor of period d if and only if $A \in \Lambda_{\text {final }}^{R}(f)$, A has d connected components and there is an open set $U \subseteq X$ such that $U \cap R_{\text {final }}(f)=A$.

(3) Each attractor admits a fundamental system of isolating neighbourhoods.

Proof. Item (1) follows by the same argument used in Proposition 5.6.

It is clear that an attractor of period $d$ is an isolated $R$-final class with $d$ connected components. Conversely, take $A \in \Lambda_{\text {final }}^{R}(f)$ with $d$ connected components, isolated in $R_{\text {final }}(f)$. Then, one can easily checks that the connected components $A_{0}, A_{1}, \cdots, A_{d-1}$ of $A$ are cyclically permuted by $f$. Let $D=R_{\text {final }}(f) \backslash A$. Then $D$ is closed since $A$ is isolated. Choose $\gamma>0$ such that the open balls $B_{\gamma}(D)$, and $B_{\gamma}\left(A_{i}\right)$, for $i=$ $0,1, \cdots, d-1$, are pairwise disjoint. Because $\left\{\Omega\left(f_{\epsilon}^{*}\right)\right\}_{\epsilon>0}$ is an outer approximation of $R(f)$ we can take $\epsilon_{0}>0$ such that $\Omega\left(f_{\epsilon}^{*}\right) \subseteq B_{\gamma}(R(f))$, for all $0<\epsilon<\epsilon_{0}$. Since $A \succ_{f} D$ we can make $\epsilon_{0}>0$ smaller, but positive, so that $A \not \psi_{f_{\epsilon}^{*}} D$ for every $0<\epsilon<\epsilon_{0}$. 
Let $U_{i}^{\epsilon}$ be the connected component of $\Omega\left(f_{\epsilon}^{*}\right)$ that contains $A_{i}(0 \leq i \leq d-1)$. Of course for $0<\epsilon<\epsilon_{0}, \quad U_{i}^{\epsilon}$ is an $\Omega$-final class in $\Sigma_{\text {final }}^{\Omega}\left(f_{\epsilon}^{*}\right)$. Then $A_{i} \subseteq U_{i}^{\epsilon} \subseteq B_{\gamma}\left(A_{i}\right)$, which implies that $A_{i} \subseteq \cap_{\epsilon>0} U_{i}^{\epsilon} \subseteq B_{\gamma}\left(A_{i}\right) \cap R(f)$. On the other hand, the fact that each $U_{i}^{\epsilon}$ is a final class easily implies that the nested intersection $\cap_{\epsilon>0} U_{i}^{\epsilon}$ is contained in $B_{\gamma}\left(A_{i}\right) \cap R_{\text {final }}(f)=A_{i}$. Therefore $\left\{U_{i}^{\epsilon}\right\}_{\epsilon>0}$ is an outer approximation of $A_{i}$. Because $U_{i}^{\epsilon}$ is $\left(f_{\epsilon}^{*}\right)^{d}$-fully invariant it follows that

$$
\overline{f^{d}\left(U_{i}^{\epsilon}\right)} \subseteq\left(f_{\epsilon}^{*}\right)^{d}\left(U_{i}^{\epsilon}\right)=U_{i}^{\epsilon}
$$

Therefore, $\left\{U_{i}^{\epsilon}\right\}_{\epsilon>0}$ is a fundamental system of isolating neighbourhoods for $A_{i}$. Clearly

$$
A_{i}=f^{d}\left(A_{i}\right) \subseteq \bigcap_{n=1}^{\infty} f^{n d}\left(U_{i}^{\epsilon}\right) .
$$

On the other hand, for any $0<\delta<\epsilon_{1}<\epsilon$,

$$
A_{i} \subseteq \bigcap_{n=1}^{\infty} f^{n d}\left(U_{i}^{\epsilon}\right) \subseteq \bigcap_{n=1}^{\infty}\left(f_{\delta}^{*}\right)^{n d}\left(U_{i}^{\epsilon}\right) \subseteq U_{i}^{\epsilon_{1}}
$$

where last inclusion follows by Proposition 5.8. Since $0<\epsilon_{1}<\epsilon$ is arbitrary small, it follows that $A_{i}=\bigcap_{n=1}^{\infty} f^{n d}\left(U_{i}^{\epsilon}\right)$ is a cyclic attractor of period $d$.

Lemma 6.2. Given $f \in \mathcal{C}^{0}(X)$,

$$
R_{\text {final }}(f)=\varlimsup_{\epsilon \rightarrow 0^{+}} \Omega_{\text {final }}\left(f_{\epsilon}^{*}\right):=\overline{\bigcap_{\delta>0} \cup_{0<\epsilon<\delta} \Omega_{\text {final }}\left(f_{\epsilon}^{*}\right)} .
$$

Proof. It is clear that $\left\{\Omega\left(f_{\epsilon}^{*}\right)\right\}_{\epsilon>0}$ is an outer approximation of $R(f)$. Therefore $\overline{\lim }_{\epsilon \rightarrow 0^{+}} \Omega_{\text {final }}\left(f_{\epsilon}^{*}\right) \subseteq R(f)$. Given $x \in \overline{\lim }_{\epsilon \rightarrow 0^{+}} \Omega_{\text {final }}\left(f_{\epsilon}^{*}\right)$, let $\left\{\epsilon_{n}\right\}$ be a sequence of positive numbers, decreasing to zero, such that, for every $n \in \mathbb{N}, x \in \Omega_{\text {final }}\left(f_{\epsilon_{n}}^{*}\right)$. Let us prove that $x$ is a final chain recurrent state. Assume $x \rightarrow_{f} y$. Then for all large enough $n \in \mathbb{N}, x \rightsquigarrow f_{\epsilon_{n}}^{*} y$, which implies, because $x$ is a final recurrent state for $f_{\epsilon_{n}}^{*}$, that $y \rightsquigarrow f_{\epsilon_{n}}^{*} x$. Since $\epsilon_{n}>0$ is arbitrary small, it follows that $y \rightarrow_{f} x$. Thus $x \in R_{\text {final }}(f)$.

Now, assume that $x \notin \varlimsup_{\epsilon \rightarrow 0^{+}} \Omega_{\text {final }}\left(f_{\epsilon}^{*}\right)$, and let us prove that $x \notin R_{\text {final }}(f)$. For some $\delta>0, x \notin A_{\delta}=\overline{\bigcup_{0<\epsilon<\delta} \Omega_{\text {final }}\left(f_{\epsilon}^{*}\right)}$. By Proposition 5.3 we can take, for each $0<\epsilon<\delta, z_{\epsilon} \in \Omega_{\text {final }}\left(f_{\epsilon}^{*}\right) \cap W_{\Omega}^{\mathrm{s}}\left(x, f_{\epsilon}^{*}\right)$. Let $z$ be a sublimit of $\left\{z_{\epsilon}\right\}_{\epsilon>0}$. Then $x \rightarrow_{f} z$. On the other hand $z \psi_{f_{\epsilon}^{*}} x$, because $x \notin A_{\delta}$ for each $0<\epsilon<\delta$. This proves that $x$ is not a final state. Therefore $x \notin R_{\text {final }}(f)$. 
Proposition 6.3. Given $f \in \mathcal{C}^{0}(X), X=W_{R}^{\mathrm{u}}\left(R_{\text {final }}(f)\right)$.

Proof. Given $x \in X$, take for each $\epsilon>0 \quad z_{\epsilon} \in \Omega_{\text {final }}\left(f_{\epsilon}^{*}\right)$ such that $x \rightsquigarrow_{f_{\epsilon}^{*}} z_{\epsilon}$. Let $z$ be a sublimit of $\left\{z_{\epsilon}\right\}_{\epsilon>0}$. Then by the previous proposition $z \in R_{\text {final }}(f)$. It is also clear that $x \rightarrow_{f} z$. Thus $x \in W_{R}^{\mathrm{u}}\left(R_{\text {final }}(f)\right)$.

A connected component of $R_{\text {final }}(f)$ will be called an $R$-final component of $f$. We denote by $\Sigma_{\text {final }}^{R}(f)$ the quotient set of all $R$-final components of $f$. Unlike the case of open maps, the set $\Sigma_{\text {final }}^{R}(f)$ may be infinite. Nevertheless, the mapping $f: X \rightarrow X$ induces an action on the quotient $\bar{\pi}_{f}: \Sigma_{\text {final }}^{R}(f) \rightarrow \Sigma_{\text {final }}^{R}(f)$, defined by $\bar{\pi}_{f}(C)=f(C)$,

We say that a continuous mapping $f: X \rightarrow X$ is weakly combinatorially stable if and only if there is $\epsilon>0$ such that all $\varphi \in \mathcal{O}(X)$ satisfying

(1) $f \prec \varphi$, i.e. $\operatorname{graph}(f) \subseteq \operatorname{graph}(\varphi)$, and

(2) $\operatorname{diam}(\varphi)=\sup _{x \in X} \operatorname{diam}(\varphi(x))<\epsilon$

are combinatorially equivalent to $f$. We say that $\varphi$ is combinatorially equivalent to $f$ if and only if there is a bijective map $h: \sum_{\text {final }}^{\Omega}(\varphi) \rightarrow \Sigma_{\text {final }}^{R}(f)$ which conjugates the action of $\pi_{\varphi}$ on $\Sigma_{\text {final }}^{\Omega}(\varphi)$ with that of $\bar{\pi}_{f}$ on $\sum_{\text {final }}^{R}(f)$, i.e. $\quad \bar{\pi}_{f} \circ h=h \circ \pi_{\varphi}$.

Theorem 6.1. Given $f \in \mathcal{C}^{0}(X)$, the following statements are equivalent:

(1) $f$ has finitely many attractors;

(2) $\Sigma_{\text {final }}^{R}(f)$ is a finite set;

(3) $f$ is weakly combinatorially stable.

Proof. $(2) \Rightarrow(1)$. Assume that $\Sigma_{\text {final }}^{R}(f)$ is finite. Then every class $C \in \Lambda_{\text {final }}^{R}(f)$ is isolated with finitely many connected components, and, by Proposition 6.1, is a periodic attractor of $f$. By Proposition 6.3, the pseudo-basins of these attractors cover all $X$. Therefore $f$ has finitely many attractors.

$(1) \Rightarrow(2)$. This is obvious.

$(2) \Rightarrow(3)$. Assume that $\Sigma_{\text {final }}^{R}(f)$ is finite. For each $\epsilon>0$ we can define, as in the previous section, $\theta_{f, f_{\epsilon}^{*}}^{*}: \Sigma_{\text {final }}^{\Omega}\left(f_{\epsilon}^{*}\right) \rightarrow \mathcal{P}\left(\Sigma_{\text {final }}^{R}(f)\right)$ to be the map that to each class $C \in \Sigma_{\text {final }}^{\Omega}\left(f_{\epsilon}^{*}\right)$ assigns the set of $R$-final classes in $\Sigma_{\text {final }}^{R}(f)$ contained in $C$. With our finitness assumption we can prove, as in Proposition 5.16, that for some $\epsilon>0, \quad \theta_{f, f_{\epsilon}^{*}}^{*}$ 
is a final equivalence. Using the factorization Lemma 5.14 we see that $\theta_{\varphi, f_{\epsilon}^{*}}$ is a final equivalence for each open map $\varphi \in \mathcal{O}(X)$ with $f \prec \varphi \prec f_{\epsilon}^{*}$. Therefore $f$ is weakly combinatorially stable.

$$
\text { (3) } \Rightarrow(2) \text {. Clear. }
$$

We mention two examples of continuous maps $f \in \mathcal{C}^{0}(X)$ which are not weakly combinatorially stable. First, we have maps with infinitely many attractors. They appear associated to Newhouse phenomenon of persistent heteroclinic tangencies in some hyperbolic invariant set. These classes of maps are residual in open sets of systems, with respect to the $C^{2}$ topology. Second we have infinitely renormalizable maps. Such examples admit totally disconnected 'attractors' and, therefore, can not be weakly combinatorially stable. Of course weak combinatorial stability can not be $C^{2}$ generic, as Newhouse examples show. But according to the Palis conjecture this weak combinatorial stability should be dense in every space $\mathcal{C}^{r}(X)$ with the $C^{r}$ topology.

Theorem 6.2. The set of maps $f \in \mathcal{C}^{0}(X)$ with finitely many attractors is dense in $\mathcal{C}^{0}(X)$.

The rest of this section is devoted to prove this theorem. Given $f \in \mathcal{C}^{0}(X)$ and $\epsilon>0$ we will denote by $f_{\epsilon}^{\bullet}$ the open map defined by $f_{\epsilon}^{\bullet}(x)=B_{\epsilon}(f(x))$, where the left hand side stands for the geodesic ball of radius $\epsilon>0$. Since we are dealing with continuous mappings, $\left\{f_{\epsilon}^{\bullet}\right\}_{\epsilon>0}$ is an outer approximation of $f$. By convention we set $f_{0}^{\bullet}=f$. Comparing with the standard outer approximation note that $f \prec f_{\epsilon}^{\bullet} \leq f_{\epsilon}^{*}$.

Given a finite subset $Z \subseteq X$, we will call skeleton-chain to any map $\sigma: Z \rightarrow \mathcal{P}(Z)$ such that for every $x \in Z$ there is some $n \geq 1$ such that $\sigma^{n}(x)=\varnothing$. States $x \in Z$ such that $\sigma(x)=\varnothing$ will be called terminal states. Similarly, when $\sigma^{-1}(x)=\varnothing, x \in Z$ will be called a source state. Clearly, a skeleton-chain is essentially a directed graph with no cycles. Consider open maps $\varphi, \psi \in \mathcal{O}(X)$. When a skeleton-chain $\sigma$ has all its terminal and source states in $\Omega_{\text {final }}(\varphi)$ we will call it a skeleton-chain over $\varphi$. We say that a finite sequence $\left\{x_{0}, x_{1}, \cdots, x_{m}\right\}$ of points in $Z$ is a $\sigma$-chain over $\varphi$ if and only if it starts and finishes at points $x_{0}, x_{m} \in \Omega_{\text {final }}(\varphi)$ and for every $i=1, \cdots, m$, $x_{i} \in \sigma\left(x_{i-1}\right)$. A skeleton-chain over $\varphi$ can be seen as a finite set of $\sigma$-chains over $\varphi$ which may cross each other, but without forming cycles. We will say that a skeleton-chain $\sigma$ 
is subordinated to $\psi$ if $\sigma(x) \subseteq \psi(x)$ for all $x \in Z$. We then write $\sigma \prec \psi$ to express the fact that $\sigma$ is subordinated to $\psi$. Given two skeleton-chains over $\varphi, \sigma_{1}: Z_{1} \rightarrow \mathcal{P}\left(Z_{1}\right)$ and $\sigma_{2}: Z_{2} \rightarrow \mathcal{P}\left(Z_{2}\right)$, we say that $\sigma_{1}$ is epimorphic (over $\varphi$ ) to $\sigma_{2}$ if and only if there is a surjective map $h: Z_{1} \rightarrow Z_{2}$ such that $h^{-1}\left(\sigma_{2}(x)\right) \subseteq \sigma_{1}\left(h^{-1}(x)\right)$ for every $x \in Z_{2}$, and all states in $h^{-1}(x) \cup\{x\}$ belong to the same connected component of $\Omega_{\text {final }}(\varphi)$, for every terminal and source states $x \in Z_{2}$. This easily implies that every $\sigma_{2}$-chain over $\varphi$ can be lifted into a $\sigma_{1}$-chain over $\varphi$ with the same length, which starts and finishes exactly at the same components of $\Omega_{\mathrm{final}}(\varphi)$.

Definition 6.1. Let $\mathcal{S} \subseteq \mathcal{C}^{0}(X)$ be a class of continuous mappings. We say that $\mathcal{S}$ has the skeleton-chain perturbation property if and only if:

(1) $\mathcal{S}$ is closed with respect to the metric $d_{H}$;

(2) given $0<r_{0}<r$ there is $\epsilon>0$ such that for every $f \in \mathcal{S}$ and any skeleton-chain $\sigma$ over $f_{r_{0}}^{\bullet}$ subordinated to $f_{r+\epsilon}^{\bullet}$, there is some $g \in \mathcal{S}$ such that $f_{r_{0}}^{\bullet} \prec g_{r}^{\bullet} \prec f_{r+\epsilon}^{\bullet}$, and a skeleton-chain $\sigma^{\prime}$ over $f_{r_{0}}^{\bullet}$ epimorphic to $\sigma$ which is subordinated to $g_{r}^{\bullet}$.

Theorem 6.2 is a corollary of Theorem 6.3 and Proposition 6.4 bellow.

Theorem 6.3. Let $\mathcal{S} \subseteq \mathcal{C}^{0}(X)$ be a class of continuous mappings with the skeletonchain perturbation property. Given $f \in \mathcal{S}$ and $\epsilon>0$ there is some $g \in \mathcal{S}$ such that $g \prec f_{\epsilon}^{\bullet}$ and $g$ is combinatorially equivalent to $f_{\epsilon}^{\bullet}$. In particular, $g$ has finitely many attractors.

Proposition 6.4. The class $\mathcal{S}=\mathcal{C}^{0}(X)$ has the skeleton-chain perturbation property.

Consider now any class $\mathcal{S} \subseteq \mathcal{C}^{0}(X)$ of continuous mappings with the skeleton-chain perturbation property and define

$$
\mathcal{X}=\left\{f_{\epsilon}^{\bullet}: f \in \mathcal{S}, \epsilon \geq 0\right\} .
$$

This space can be naturally identified with the product $\mathcal{S} \times[0,+\infty[$ through the correspondence $f_{\epsilon}^{\bullet} \equiv(f, \epsilon)$. With this identification in mind consider the projection mapping $\delta: \mathcal{X} \rightarrow\left[0,+\infty\left[\right.\right.$, defined by $\delta\left(f_{\epsilon}^{\bullet}\right)=\epsilon$. For each $\varphi \in \mathcal{X}, \delta(\varphi)$ will be called the radius of $\varphi$. Finally we define $\mathcal{X}^{\text {stable }}$ to be the set of all open maps $\varphi \in \mathcal{X}$ such that either $\delta(\varphi)=0$ or else $\varphi$ satisfies the combinatorial stability condition. Clearly, given any $f \in \mathcal{S}, \quad f_{\epsilon}^{\bullet} \in \mathcal{X}^{\text {stable }}$ for all but a countable set of parameters $\epsilon>0$. 
Definition 6.2. A mapping $F: \mathcal{X}^{\text {stable }} \rightarrow \mathcal{X}^{\text {stable }}$ is called a compression operator if and only if $F(\varphi)=\varphi \Leftrightarrow \delta(\varphi)=0$, and whenever $\delta(\varphi)>0$

(1) $F(\varphi) \prec \varphi$;

(2) $0<\delta(F(\varphi))<\delta(\varphi)$; and

(3) $F(\varphi) \bowtie \varphi$, i.e. $F(\varphi)$ and $\varphi$ are combinatorially equivalent.

We will denote by $\mathcal{M}$ the set of all compression operators on $\mathcal{X}^{\text {stable }}$.

Proposition 6.5. $\mathcal{M} \neq \varnothing$, i.e. there are compression operators on $\mathcal{X}^{\text {stable }}$.

Proof. Given $\varphi \in \mathcal{X}^{\text {stable }}$ let $\epsilon(\varphi)$ be the greatest lower bound of all $\epsilon>0$ given by Proposition 5.17. Let then $\mathcal{G}(\varphi)=\left\{\psi \in \mathcal{X}: \varphi_{\epsilon}^{\circ} \prec \psi \prec \varphi\right.$ with $\left.\epsilon=\epsilon(\varphi)\right\}$. Clearly, any selection function $F: \mathcal{X}^{\text {stable }} \rightarrow \mathcal{X}$ with $F(\varphi) \in \mathcal{G}(\varphi)$ for all $\varphi \in \mathcal{X}^{\text {stable }}$, is a compression operator. Therefore, by the Axiom of Choice, there are compression operators.

Each compression operator $F \in \mathcal{M}$ will define a kind of dynamical system with discrete time modeled by the first uncountable ordinal $\Omega$. For each ordinal $\alpha<\Omega$, the power $F^{\alpha}$ will be defined as a compression operator in $\mathcal{M}$. We shall see that radius function $\delta: \mathcal{X}^{\text {stable }} \rightarrow[0,+\infty[$ is some kind of Lyapounov function decreasing along orbits of $F$. More precisely, we shall see that, given any $\varphi \in \mathcal{X}^{\text {stable }}$, the sequence $\left\{\delta\left(F^{\alpha}(\varphi)\right)\right\}_{\alpha<\Omega}$ decreases and is eventually constant equal to zero, which proves that $\left\{F^{\alpha}(\varphi)\right\}_{\alpha<\Omega}$ is eventually constant equal to some function $f \in \mathcal{S}$. It follows easily that $f \prec \varphi$ is combinatorially equivalent to $\varphi$. In particular $f$ will have finitely many attractors. Let $\omega$ denote the first infinite ordinal, $\omega=\{0,1,2, \cdots\}(\equiv \mathbb{N})$. Note that any ordinal $n<\omega$ is a natural number and so we can define $F^{n}$ to be the usual $n$-fold composition $F^{n}=F \circ \cdots \circ F$. The following proposition is the key to define these 'dynamical systems' and prove Theorem 6.3. The assumption that $\mathcal{S}$ has the skeleton-chain perturbation property is used in the proof of this proposition.

Proposition 6.6. Given $F \in \mathcal{M}$ there is another compression operator $F^{\omega} \in \mathcal{M}$ such that

$$
\delta\left(F^{\omega}(\varphi)\right) \leq \inf _{n<\omega} \delta\left(F^{n}(\varphi)\right) .
$$


This crucial fact will be proved in the end of this section. Referring to the power $F^{\omega}$ of an operator $F \in \mathcal{M}$, we now define recursively all powers $F^{\alpha}$, where $\alpha<\Omega$ is any countable ordinal. More precisely we shall define operators $\operatorname{Pow}_{\alpha}: \mathcal{M} \rightarrow \mathcal{M}$, one for each ordinal $\alpha<\Omega$.

Definition 6.3. For $n<\omega, \operatorname{Pow}_{n}(F)=F^{n}$ is the usual $n$-fold composition. $\operatorname{Pow}_{\omega}$ is an operator, that can be defined according to Proposition 6.6. Given any $\omega<\alpha<\Omega$, if $\alpha$ is not a limit ordinal then $\alpha=\beta+1$, and we define

$$
\operatorname{Pow}_{\alpha}(F)=F \circ \operatorname{Pow}_{\beta}(F)=F \circ F^{\beta} .
$$

Otherwise if $\alpha$ is a limit ordinal then $\alpha=\omega \cdot \beta=\overbrace{\omega+\omega+\cdots}^{\beta \text { times }}$ for some ordinal $\beta<\alpha$ and we define

$$
\operatorname{Pow}_{\alpha}(F)=\operatorname{Pow}_{\beta}\left(\operatorname{Pow}_{\omega}(F)\right)=\left(F^{\omega}\right)^{\beta} .
$$

A sequence $\left\{r_{\alpha}\right\}_{\alpha<\Omega}$, of non-negative real numbers $r_{\alpha} \geq 0$, is said to be strictly decreasing if and only if $r_{\alpha} \leq \inf _{\beta<\alpha} r_{\beta}$ whenever $\alpha$ is a limit ordinal, and $r_{\alpha+1}<r_{\alpha}$ whenever $r_{\alpha}>0$. The sequence $\left\{r_{\alpha}\right\}_{\alpha<\Omega}$ is said to be eventually zero if and only if there is some ordinal $\gamma<\Omega$ such that $r_{\alpha}=0$ for all $\alpha \geq \gamma$.

Lemma 6.7. Every strictly decreasing sequence $\left\{r_{\alpha}\right\}_{\alpha<\Omega}$, of non-negative real numbers $r_{\alpha} \geq 0$, is eventually zero.

Proof. For each integer $n \geq 1$, the set $A_{n}=\left\{\alpha<\Omega: r_{\alpha}-r_{\alpha+1} \geq 1 / n\right\}$ is finite. Therefore $\alpha_{n}=\cup A_{n}<\Omega$ is a countable ordinal. Since $\alpha_{1} \leq \alpha_{2} \leq \cdots \alpha_{n} \leq \cdots$ is a countable sequence of countable ordinals, $\gamma=\cup_{n=1}^{\infty} \alpha_{n}$ is again a countable ordinal. Clearly $r_{\alpha}=0$ for every $\alpha \geq \gamma$.

Proposition 6.8. For every $\varphi \in \mathcal{X}^{\text {stable }}$ and $F \in \mathcal{M}$, the sequence $\left\{\delta\left(F^{\alpha}(\varphi)\right)\right\}_{\alpha<\Omega}$ is strictly decreasing and, therefore, it is eventually zero.

Proof. Given $\alpha<\Omega$ such that $\delta\left(F^{\alpha}(\varphi)\right)>0$, since $F^{\alpha+1}(\varphi)=F\left(F^{\alpha}(\varphi)\right)$, by item (2) in the definition of a compression operator, it follows that $\delta\left(F^{\alpha+1}(\varphi)\right)>0$. Let us prove, by transfinite induction in $\alpha<\Omega$, that

$$
\left(P_{\alpha}\right) \text { for all } \beta<\alpha, \varphi \in \mathcal{X}^{\text {stable }} \text { and } F \in \mathcal{M}, \delta\left(F^{\alpha}(\varphi)\right) \leq \delta\left(F^{\beta}(\varphi)\right) .
$$


If $\alpha=n$ is a finite ordinal it follows by Definition 6.2 that a strict inequality holds. Proposition 6.6 shows that $\left(P_{\omega}\right)$ holds.

Assume now that $\left(P_{\beta}\right)$ holds for all $\beta<\alpha$ and let us prove that $\left(P_{\alpha}\right)$ is also true. Suppose $\alpha$ is a limit ordinal. Otherwise $\left(P_{\alpha}\right)$ is trivial. Then there is some $\alpha_{1}<\alpha$ such that $\alpha=\omega \cdot \alpha_{1}$. Now, given $\beta<\alpha$ there are $\beta_{1}<\beta$ and $n<\omega$ such that $\beta=\omega \cdot \beta_{1}+n$. To prove this define $\beta_{2}=\min \left\{\beta^{\prime} \leq \beta: \exists n<\omega \beta=\beta^{\prime}+n\right\}$. Then $\beta=\beta_{2}+n$ and $\beta_{2}$ is a limit ordinal. Therefore $\beta_{2}=\omega \cdot \beta_{1}$ for some $\beta_{1}<\beta_{2} \leq \beta$. Since $\alpha$ is a limit ordinal $\omega \cdot \beta_{1}+n=\beta<\alpha$ implies that $\omega \cdot\left(\beta_{1}+1\right)=\omega \cdot \beta_{1}+\omega \leq \alpha=\omega \cdot \alpha_{1}$, which in turn implies that $\beta_{1}+1 \leq \alpha_{1}$. Thus

$$
\begin{aligned}
\delta\left(F^{\alpha}(\varphi)\right) & =\delta\left(\left(F^{\omega}\right)^{\alpha_{1}}(\varphi)\right) \leq \delta\left(\left(F^{\omega}\right)^{\beta_{1}+1}(\varphi)\right) \\
& =\delta\left(F^{\omega}\left(\left(F^{\omega}\right)^{\beta_{1}}(\varphi)\right)\right) \leq \delta\left(F^{n}\left(F^{\omega \cdot \beta_{1}}(\varphi)\right)\right)=\delta\left(F^{\beta}(\varphi)\right)
\end{aligned}
$$

proving $\left(P_{\alpha}\right)$.

Proof. (of Theorem 6.3) Given $f \in \mathcal{S}$ and $\epsilon>0$, taking $\epsilon$ smaller if necessary, we may assume that $\varphi=f_{\epsilon}^{\bullet} \in \mathcal{X}^{\text {stable }}$. Consider the transfinite sequence $\left\{F^{\alpha}(\varphi)\right\}_{\alpha<\Omega}$. Let $\gamma<\Omega$ be the first ordinal such that $\delta\left(F^{\gamma}(\varphi)\right)=0$. Then $h=F^{\gamma}(\varphi) \in \mathcal{S}$ is a continuous map such that $h \prec \varphi$ and $h$ is combinatorially equivalento to $\varphi$, which proves the theorem.

Two simple lemmas are needed to prove Proposition 6.6.

Lemma 6.9. $f_{r}^{\bullet} \prec g_{s}^{\bullet} \Leftrightarrow d_{H}(f, g)<s-r$.

Next lemma states that in an open map extension $\varphi_{0} \prec \varphi_{1}$, the combinatorics of $\varphi_{1}$ missing in $\varphi_{0}$ can be encapsulated in some skeleton-chain $\sigma$ over $\varphi_{0}$ which is subordinated to $\varphi_{1}$.

Lemma 6.10. Given open maps $\varphi_{0}, \varphi_{1} \in \mathcal{O}_{\text {Cont }}(X)$ with $\varphi_{0} \prec \varphi_{1}$, there is a skeletonchain $\sigma$ over $\varphi_{0}$, subordinated to $\varphi_{1}$, such that all open maps $\varphi_{0} \leq \psi \leq \varphi_{1}$, admiting a skeleton-chain $\sigma^{\prime}$ over $\varphi_{0}$ epimorphic to $\sigma$, are combinatorially equivalent to $\varphi_{1}$.

Proof. (of Proposition 6.6) Take any operator $F \in \mathcal{M}$. Given $\varphi \in \mathcal{X}^{\text {stable }}$, let $\varphi_{n}=\left(f_{n}\right)_{\delta_{n}}^{\bullet}$ be the sequence $\varphi_{n}=F^{n}(\varphi)$. The sequence $\left\{\delta_{n}\right\}$ is strictly decreasing and we set $r=\lim _{n \rightarrow \infty} \delta_{n}$. The sequence of maps $\left\{f_{n}\right\}$ is Cauchy because, by Lemma 6.9 $\left(f_{n+1}\right)_{\dot{\delta}_{n+1}}^{\bullet} \prec\left(f_{n}\right)_{\delta_{n}}^{\bullet}$ implies $d_{H}\left(f_{n}, f_{n+1}\right)<\delta_{n}-\delta_{n+1}$, and the series $\sum_{n \geq 1} \delta_{n}-\delta_{n+1}$ is 
summable. Since $\mathcal{S}$ is complete we can take then $f \in \mathcal{S}$ such that $d_{H}\left(f_{n}, f\right) \rightarrow 0$ as $n \rightarrow \infty$. In order to define $F^{\omega}(\varphi)$ we consider two cases.

First case: $r=0$. In this case we define $F^{\omega}(\varphi)=f$. Clearly $f \prec \varphi$ and $f$ is combinatorially equivalent to $\varphi$.

Second case: $r>0$. In this case take $0<r_{0}<r$, as in Proposition 5.17, such that $f_{r_{0}}^{\bullet}$ is combinatorially equivalent to $f_{r}^{\bullet}$. Note that it may happen that $f_{r}^{\bullet}$ is not combinatorially stable, and so not combinatorially equivalent to any $\varphi_{n}$. For the pair of positive real numbers $0<r_{0}<r$ we can, according to Definition 6.1 , take $\epsilon>0$ in order to fulfill the second requirement of this definition. Then we have $\varphi_{n} \prec f_{r+\epsilon}^{\bullet}$, for all large enough $n$. Taking, if necessary, $\epsilon>0$ smaller we can assume that $\varphi_{n} \prec f_{r+\epsilon}^{\bullet} \prec \varphi$. In particular, these three open maps are combinatorially equivalent. Choose now a skeleton-chain $\sigma$ over $f_{r_{0}}^{\bullet}$ subordinated to $f_{r+\epsilon}^{\bullet}$, as given in Lemma 6.10. Then by definition of the 'skeleton-chain perturbation property', see Definition 6.1, there is some map $g \in \mathcal{S}$ such that $f_{r_{0}}^{\bullet} \prec g_{r}^{\bullet} \prec f_{r+\epsilon}^{\bullet}$. There is also some chain skeleton $\sigma^{\prime}$ over $f_{r_{0}}^{\bullet}$, epimorphic to $\sigma$ over $f_{r_{0}}^{\bullet}$, and subordinated to $g_{r}^{\bullet}$. Lemma 6.10 above now implies that $g_{r}^{\bullet}$ is combinatorially equivalent to $f_{r+\epsilon}^{\bullet}$ and, therefore, to $\varphi$. In this case we define $F^{\omega}(\varphi)=g_{r}^{\bullet}$.

Finally to prove Proposition 6.4 we shall use the following lemma.

Lemma 6.11. Given $f \in \mathcal{C}^{0}(X), Z \subseteq X$ finite, and $\epsilon>\delta>0$, there is some $g \in \mathcal{C}^{0}(X)$ with $d_{H}(f, g)<\epsilon$ such that $g\left(B_{\delta}(x)\right) \supseteq \bar{B}_{\epsilon-\delta}(f(x)), \quad \forall x \in Z$.

Proof. Take $0<\eta<\delta$ so that the oscillation of $f$ in each ball $B_{\eta}(x)$, with $x \in Z$, is less than $\delta>0$. Then any map $g \in \mathcal{C}^{0}(X)$ which coincides with $f$ outside $B_{\eta}(Z)$, and takes values in $\bar{B}_{\epsilon-\delta}(f(x))$ for arguments in $B_{\eta}(x)$, must satisfy $d_{H}(f, g)<\epsilon$. Of course we can choose such a $g$ in a way that $g\left(B_{\eta}(x)\right)$ covers $\bar{B}_{\epsilon-\delta}(f(x))$, for each $x \in Z$.

Proof. (of Proposition 6.4) We only have to prove that $\mathcal{S}=\mathcal{C}^{0}(X)$ satisfies Definition 6.1(2). Given $0<r_{0}<r$ take any $0<\epsilon<r-r_{0}$. Take $f \in \mathcal{C}^{0}(X)$, and let $\sigma: Z \rightarrow \mathcal{P}(Z)$ be a skeleton-chain over $f_{r_{0}}^{\bullet}$ subordinated to $f_{r+\epsilon}^{\bullet}$. Choose then $0<\delta<\epsilon / 2$ such that:

(a) $B_{\delta}\left(Z \cap \Omega_{\text {final }}\left(f_{r_{0}}^{\bullet}\right)\right) \subseteq \Omega_{\text {final }}\left(f_{r_{0}}^{\bullet}\right)$,

(b) $\operatorname{graph}(\sigma) \subseteq \operatorname{graph}\left(f_{r+\epsilon-2 \delta}^{\bullet}\right)$, 
and take $g \in \mathcal{C}^{0}(X)$, according to Lemma 6.11, such that $d_{H}(f, g)<\epsilon$ and, for all $x \in Z, g\left(B_{\delta}(x)\right) \supseteq \bar{B}_{\epsilon-\delta}(f(x))$. Since $d_{H}(f, g)<\epsilon<r-r_{0}$, by Lemma 6.9 , this implies that $f_{r_{0}}^{\bullet} \prec g_{r}^{\bullet} \prec f_{r+\epsilon}^{\bullet}$. Let us prove now that there is a skeleton-chain $\sigma^{\prime}$ over $f_{r_{0}}^{\bullet}$ epimorphic to $\sigma$ and subordinated to $g_{r}^{\bullet}$. This will be proved by induction. To set up the induction scheme define $Z_{0}=\{x \in Z: \sigma(x)=\varnothing\}$ and, for each $n \geq 1$, $Z_{n}=\left\{x \in Z: \sigma(x) \subseteq Z_{n-1}\right\}$. Clearly, for some $N \in \mathbb{N}, Z_{0} \subset Z_{1} \subset \cdots \subset Z_{N}=Z$ and for each $0 \leq n \leq N, \quad \sigma_{n}=\sigma_{\mid Z_{n}}$ is a skeleton-chain on $Z_{n}$. Define $Z_{0}^{\prime}=Z_{0}$, $h_{0}: Z_{0}^{\prime} \rightarrow Z_{0}$ to be the identity map, and $\sigma_{0}^{\prime}=\sigma_{0}$. Assume, as our induction hypothesis, that there is a surjective map $h_{n-1}: Z_{n-1}^{\prime} \rightarrow Z_{n-1}$ such that for all $x \in Z_{n-1}, \quad(1) d\left(h_{n-1}^{-1}(x), x\right)<\delta$, and $(2) h_{n-2}^{-1}(\sigma(x))=\sigma_{n-1}^{\prime}\left(h_{n-1}^{-1}(x)\right)$. Let us construct now $Z_{n}^{\prime}, \sigma_{n}^{\prime}$ and $h_{n}: Z_{n}^{\prime} \rightarrow Z_{n}$, surjective, satisfying the correspondent conditions $\left(1^{\prime}\right) d\left(h_{n}^{-1}(x), x\right)<\delta$, and $\left(2^{\prime}\right) h_{n-1}^{-1}(\sigma(x))=\sigma_{n}^{\prime}\left(h_{n}^{-1}(x)\right)$, for every $x \in Z_{n}$. Take $x \in Z_{n}$ and let $\sigma(x)=\left\{y_{1}, \cdots, y_{m}\right\} \subseteq Z_{n-1}$. For each $1 \leq i \leq m$, consider the pre-images $\left(h_{n-1}\right)^{-1}\left(y_{i}\right)=\left\{y_{i, 1}^{\prime}, y_{i, 2}^{\prime}, \cdots, y_{i, k_{i}}^{\prime}\right\}$, with $k_{i} \geq 1$. Thus

$$
\left(h_{n-1}\right)^{-1}(\sigma(x))=\left\{y_{1,1}^{\prime}, \cdots, y_{1, k_{1}}^{\prime}, y_{2,1}^{\prime}, \cdots, y_{2, k_{2}}^{\prime}, \cdots, y_{m, 1}^{\prime}, \cdots, y_{i, k_{m}}^{\prime}\right\}
$$

and, by item (1) of the induction hypothesis, each $y_{i, j}^{\prime}$ in this set satisfies $d\left(y_{i, j}^{\prime}, y_{i}\right)<$ $\delta$. Therefore, since by item (b) above $y_{i} \in \sigma(x) \subseteq f_{r+\epsilon-2 \delta}^{\bullet}(x)$,

$$
\begin{aligned}
d\left(y_{i, j}^{\prime}, f(x)\right) & \leq d\left(y_{i, j}^{\prime}, y_{i}\right)+d\left(y_{i}, f(x)\right) \\
& \leq \delta+r+\epsilon-2 \delta=r+\epsilon-\delta,
\end{aligned}
$$

and so

$$
y_{i, j}^{\prime} \in B_{r+\epsilon-\delta}(f(x))=B_{r}\left(\bar{B}_{\epsilon-\delta}(f(x))\right) \subseteq B_{r}\left(g\left(B_{\delta}(x)\right)\right)=g_{r}^{\bullet}\left(B_{\delta}(x)\right) .
$$

This shows that there is some $x_{i, j}^{\prime} \in B_{\delta}(x)$ such that $y_{i, j}^{\prime} \in g_{r}^{\bullet}\left(x_{i, j}^{\prime}\right)$. Clearly, this choice is not unique. In fact, there is an open set of possible choices for each $x_{i, j}^{\prime}$. Therefore, we may assume that the choice of each $x_{i, j}^{\prime}$, for every $x \in Z_{n}$, is made in such a way that they all become distinct. Now, let $Z_{n}^{\prime}(x)=\left\{x_{i, j}^{\prime}: 1 \leq i \leq m, 1 \leq j \leq k_{i}\right\}$, and define $Z_{n}^{\prime}=Z_{n-1}^{\prime} \cup \bigcup_{x \in Z_{n}} Z_{n}^{\prime}(x)$. Let $\sigma_{n}^{\prime}: Z_{n}^{\prime} \rightarrow \mathcal{P}\left(Z_{n}^{\prime}\right)$ be the extension of $\sigma_{n-1}^{\prime}$ defined by $\sigma_{n}^{\prime}\left(x_{i, j}^{\prime}\right)=y_{i, j}^{\prime}$, for every $x_{i, j}^{\prime} \in Z_{n}^{\prime}(x)$. Finally, define $h_{n}: Z_{n}^{\prime} \rightarrow Z_{n}$ extending $h_{n-1}$ in such a way that $\left(h_{n}\right)^{-1}(x)=Z_{n}^{\prime}(x)$ for every $x \in Z_{n} \backslash Z_{n-1}$. By the remark above the sets $Z_{n}^{\prime}(x)$ are pairwise disjoint, which shows that $h_{n}$ is a well-defined single-valued map. By construction $h_{n}$ is clearly surjective. We have

$$
\sigma_{n}^{\prime}\left(h_{n}^{-1}(x)\right)=\sigma_{n}^{\prime}\left(Z_{n}^{\prime}(x)\right)=\left\{y_{i, j}^{\prime}: 1 \leq i \leq m, 1 \leq j \leq k_{i}\right\}=\left(h_{n-1}\right)^{-1}(\sigma(x)),
$$


which establishes (2'). On the other hand, since $x_{i, j}^{\prime} \in B_{\delta}(x), \quad d\left(h_{n}\left(x_{i, j}^{\prime}\right), x_{i, j}^{\prime}\right)=$ $d\left(x, x_{i, j}^{\prime}\right)<\delta$, for every $x_{i, j}^{\prime} \in Z_{n}^{\prime}(x)$, which proves (1'). Thus, by induction, (1') and (2') hold for all $n$ up to $N$. Let then $Z^{\prime}=Z_{N}^{\prime}, \sigma^{\prime}=\sigma_{N}^{\prime}$ and $h=h_{N}$. By item (1') of the just proved statement we have, for all $x \in Z, d\left(h^{-1}(x), x\right)<\delta$. Let $x \in Z$ be a terminal or source state of $\sigma$. Then $x \in \Omega_{\mathrm{final}}\left(f_{r_{0}}^{\bullet}\right)$, and, by (a) above, $h^{-1}(x) \cup\{x\}$ is contained in a single connected component of $\Omega_{\text {final }}\left(f_{r_{0}}^{\bullet}\right)$. We have constructed a skeleton-chain $\sigma^{\prime}$ over $f_{r_{0}}^{\bullet}$ which is epimorphic to $\sigma$ over $f_{r_{0}}^{\bullet}$, and subordinated to $g_{r}^{\bullet}$, thus proving definition 6.1(2). 


\section{References}

[A] V. Araújo. Attractors and time averages for random maps. Ann. Inst. H. Poincaré Anal. Non Linéaire 17 (2000), 307-369.

[AF] J.-P. Aubin and H. Frankowska. Set-Valued Analysis. Birkhäuser, Boston, 2000 .

[B] P. Billingsley. Probability and Measure. Wiley Interscience, New York, 1985.

[F] K. J. Falconer. The Geometry of Fractal Sets (Cambridge Tracts in Mathematics, 85). Cambridge University Press, Cambridge, 1985.

[HPS] M.W. Hirsh, C.C. Pugh and M. Shub. Invariant Manifolds (Lecture Notes in Mathematics, 583). Springer, Berlin, 1977.

[N] S. Nadler Jr. Hyperspaces of Sets (Pure and Applied Mathematics, A Series of Monographs and Textbooks). Marcel Dekker, New York, 1978.

[P] J. Palis. A global view of dynamics and a conjecture on the denseness of finitude of attractors. Astérisque 261 (2000), 335-347.

[Pi] S. Yu. Pilyugin. The Space of Dynamical Systems with the $C^{0}$ - topology (Lecture Notes in Mathematics, 1571). Springer, Berlin, 1994.

[S] M. Shub. Global Stability of Dynamical Systems. Springer, New York, 1987.

[Sm] S. Smale. Differentiable dynamical systems. Bull. Am. Math. Soc. 73 (1967), 747-817.

[V1] M. Viana. Stochastic Dynamics of Deterministic Systems (Lecture Notes of the XXIst Brazil Mathematics Colloquium). IMPA, 1997.

[V2] M. Viana. Dynamics: a probabilistic and geometric perspective. Proceedings of the ICM, 1998. Documenta Mathematica I (1998), 557-578. 